2011 Shanghai Asia-Pacific School and Workshop on Gravitation International Journal of Modern Physics: Conference Series

Vol. 7 (2012) 31-66

(C) World Scientific Publishing Company

DOI: $10.1142 / \mathrm{S} 2010194512004175$

\title{
AN INTRODUCTION TO LOCAL BLACK HOLE HORIZONS IN THE 3+1 APPROACH TO GENERAL RELATIVITY*
}

\author{
JOSÉ LUIS JARAMILLO \\ Max-Planck-Institut für Gravitationsphysik, \\ Albert Einstein Institut, \\ Am Mühlenberg 1, Golm D-14476, Germany \\ Jose-Luis.Jaramillo@aei.mpg.de
}

\begin{abstract}
We present an introduction to dynamical trapping horizons as quasi-local models for black hole horizons, from the perspective of an Initial Value Problem approach to the construction of generic black hole spacetimes. We focus on the geometric and structural properties of these horizons aiming, as a main application, at the numerical evolution and analysis of black hole spacetimes in astrophysical scenarios. In this setting, we discuss their dual role as an a priori ingredient in certain formulations of Einstein equations and as an a posteriori tool for the diagnosis of dynamical black hole spacetimes. Complementary to the first-principles discussion of quasi-local horizon physics, we place an emphasis on the rigidity properties of these hypersurfaces and their role as privileged geometric probes into near-horizon strong-field spacetime dynamics.
\end{abstract}

Keywords: Black holes; quasi-local horizons; Initial Value Problem.

\section{Black Holes: Global vs (Quasi-)Local Approaches}

\subsection{Establishment's picture of the gravitational collapse}

Our discussion is framed in the problem of gravitational collapse in General Relativity. The current understanding is summarized in what one could call the establishment's picture of gravitational collapse, ${ }^{1}$ a heuristic chain of results and conjectures:

(1) Singularity theorems: If gravity is able to make all light rays locally converge (namely, if trapped surfaces exist), then a spacetime singularity forms. ${ }^{2-5}$

(2) (Weak) Cosmic censorship (Conjecture): In order to preserve predictability, the formed singularity is not visible for a distant observer. ${ }^{6}$

(3) Black hole spacetimes stability (Conjecture): General Relativity gravitational dynamics drives eventually the black hole spacetime to a stationary state.

(4) Black hole uniqueness theorem: The final state is a Kerr black hole spacetime. ${ }^{7}$

*This review was originally published in Int. J. Mod. Phys. D 20 (2011) 2169-2204. 
Light bending is a manifestation of spacetime curvature and black holes constitute a dramatic extreme case of this. The standard picture of gravitational collapse above suggests two (complementary) approaches to the characterization of black holes:

(a) Global approach: (weak) cosmic censorship suggests black holes as no-escape regions not extending to infinity. Its boundary defines the event horizon $\mathscr{E}$.

(b) Quasi-local approach: Singularity theorems suggest the characterization of a black hole as a spacetime trapped region where all light rays locally converge.

The establishment's picture of gravitational collapse depicts an intrinsically dynamical scenario. Hence, a systematic methodology to the study of dynamical spacetimes is needed. We adopt an Initial (Boundary) Value Problem approach, that offers a systematic avenue to the qualitative and quantitative aspects of generic spacetimes.

\subsection{The black hole region and the event horizon}

The traditional ${ }^{5}$ approach to black holes involves global spacetime concepts, in particular a good control of the notion of infinity. Given a (strongly asymptotically predictable) spacetime $\mathcal{M}$, the black hole region $\mathscr{B}$ is defined as $\mathscr{B}=\mathcal{M}-J^{-}\left(\mathscr{I}^{+}\right)$, where $J^{-}\left(\mathscr{I}^{+}\right)$is the causal past of future null infinity $\mathscr{I}^{+}$. That is, $\mathscr{B}$ is the spacetime region that cannot communicate with $\mathscr{I}^{+}$.

We are particularly interested in characterizing a notion of boundary surface of black holes. In this global context this is provided by the event horizon $\mathscr{E}$, defined as the boundary of $\mathscr{B}$, that is $\mathscr{E}=\partial J^{-}\left(\mathscr{I}^{+}\right) \cap \mathcal{M}$. Interesting geometric and physical properties of the event horizon are: (i) $\mathscr{E}$ is a null hypersurface in $\mathcal{M}$; (ii) it satisfies an Area Theorem, ${ }^{8,9}$ so that the area of spatial sections $\mathcal{S}$ of $\mathscr{E}$ does not decrease in the evolution and, beyond that, (iii) a set of black hole mechanics laws are fulfilled. ${ }^{10}$

However, the global aspects of the event horizon also bring difficulties: (a) it is a teleological concept, i.e. the knowledge of the full (future) spacetime is needed in order to locate $\mathscr{E}$, and (b) the black hole region and the event horizon can enter into flat spacetime regions. In sum, the notion of event horizon is a too global one: it does not fit properly into the adopted Initial Value Problem approach.

\subsection{The trapped region and the trapping boundary}

The global approach requires controlling structures that are not accessible during the evolution. In this context, the seminal notion of trapped surface ${ }^{2}$ plays a crucial role, capturing the idea that all light rays emitted from the surface locally converge. Through the singularity theorems and weak cosmic censorship, it offers a benchmark for the existence of a black hole region: in strongly predictable spacetimes with proper energy conditions, trapped surfaces lie inside the black hole region. ${ }^{5}$ Moreover, their location does not involve a whole future spacetime development. 


\subsubsection{Trapped and outer trapped surfaces. Apparent horizons}

Given a closed spatial surface $\mathcal{S}$ in the spacetime, we can consider the light emitted from it along outer and inner directions given, respectively, by future null vectors $\ell^{a}$ and $k^{a}$. Then, light locally converges (in the future) at $\mathcal{S}$ if the area of the emitted light-front spheres decreases in both directions (see though Ref. 11). Denoting the area element of $\mathcal{S}$ as $d A=\sqrt{q} d^{2} x$, the infinitesimal variations of the area along $\ell^{a}$ and $k^{a}$ define outgoing and ingoing expansions $\theta^{(\ell)}$ and $\theta^{(k)}$ (see Sec. 2.1 for details)

$$
\delta_{\ell} \sqrt{q}=\theta^{(\ell)} \sqrt{q}, \quad \delta_{k} \sqrt{q}=\theta^{(k)} \sqrt{q} .
$$

A trapped surface is characterized by $\theta^{(\ell)} \theta^{(k)}>0$. In the black hole context, in which the singularity occurs in the future, we refer to $\mathcal{S}$ as a future trapped surface (TS) if $\theta^{(\ell)}<0, \theta^{(k)}<0$ and as future marginally trapped surface (MTS) if one of the expansions, say $\theta^{(\ell)}$, vanishes: $\theta^{(\ell)}=0, \theta^{(k)} \leq 0$. If a notion of naturally expanding direction for the light rays exists (e.g. in isolated systems, the outer null direction $\ell^{\mu}$ pointing to infinity), a related notion of outer trapped surface is given ${ }^{5}$ by $\theta^{(\ell)}<0$. Marginally outer trapped surfaces (MOTS) are characterized by $\theta^{(\ell)}=0$.

Before proceeding to a characterization of black holes in terms of trapped surfaces, let us consider trapped surfaces from the perspective of a spatial slice of spacetime $\Sigma$. The trapped region in $\Sigma, \mathcal{T}_{\Sigma} \subset \Sigma$, is the set of points $p \in \Sigma$ belonging to some (outer) trapped surface $\mathcal{S} \subset \Sigma$. The Apparent Horizon (AH) is then the outermost boundary of the trapped region $\mathcal{T}_{\Sigma}$. A crucial result is the following characterization $^{5,12,13}$ of AHs: if the trapped region $\mathcal{T}_{\Sigma}$ in a slice $\Sigma$ has the structure of a manifold with boundary, the $\mathrm{AH}$ is a MOTS, i.e. $\theta^{(\ell)}=0$.

Given a $3+1$ foliation of spacetime $\left\{\Sigma_{t}\right\}$, let us consider the worldtube obtained by piling up the two-dimensional AHs $\mathcal{S}_{t} \subset \Sigma_{t}$. Such an AH-worldtube does not need to be a smooth hypersurface (it is not even necessarily continuous, as discussed in Sec. 5.1.1). This is our first encounter with the notion of a spacetime worldtube foliated by MOTS. Though these worldtubes are slicing-dependent, their characterization in terms of MOTSs makes them very useful from an operational perspective.

\subsubsection{The trapped region: Definition and caveats}

From a spacetime perspective, no reference to a slice $\Sigma$ must enter into the characterization of the trapped region. The spacetime trapped region $\mathcal{T}$ is defined as the set of points $p \in \mathcal{M}$ belonging to some trapped surface $\mathcal{S} \subset \mathcal{M}$. Its boundary is referred $^{14}$ to as the trapping boundary. These concepts offer, in principle, an intrinsically quasi-local avenue to address the notion of black hole region and black hole horizon, with no reference to asymptotic quantities.

In spite of their appealing features, there are also important caveats associated with the trapped region and the trapping boundary. In particular, we lack an operational characterization of the trapping boundary (see also the contribution by 
J. M. M. Senovilla ${ }^{15}$ ). A systematic attempt to address this issue is provided by the notion of trapping horizon, ${ }^{14}$ namely smooth worldtubes of MOTS (see Sec. 2.2), as a model for the trapping boundary. Trapping horizons, that are nonunique, have led to important insights into the structure of the trapped region, though an operational characterization of the trapping boundary is still missing.

The difficulties are illustrated in the discussion of the relation between the trapping boundary and $\mathscr{E}$. In strongly predictable spacetimes with appropriate energy conditions (see, though Ref. 16), the trapped region $\mathcal{T}$ is contained in the black hole region $\mathscr{B}$. In attempts to refine this statement, support was found ${ }^{17,18}$ suggesting that the trapping boundary actually coincides with the event horizon, though later work $^{19}$ showed that the trapped region not always extends up to $\mathscr{E}$. The question is still open for (outer) trapped regions constructed on outer trapped surfaces, rather than on TSs. Important insight into these issues has been gained in recent works ${ }^{20,21}$ demonstrating truly global features of the trapped region $\mathcal{T}$. In particular:

(i) The trapping boundary cannot be foliated by MOTS.

(ii) Closed trapped surfaces can enter into the flat region. This is an important issue in this approach to black holes, since it was a main criticism in Sec. 1.2.

(iii) Closed trapped surfaces are clairvoyant, that is, they are aware of the geometry in noncausally connected spacetime regions. This nonlocal property challenges their applicability in an operational characterization of black holes.

\subsection{A pragmatic approach to quasi-local black hole horizons}

Trapping horizons offer a sound avenue towards the quasi-local understanding of black hole physics. They provide crucial insight in gravitational scenarios where a quasi-local notion of black hole horizon is essential, such as black hole thermodynamics beyond equilibrium, the characterization of physical parameters of strongly dynamical astrophysical black holes (notably in numerical simulations), semi-classical collapse, quantum gravity or mathematical relativity (cf. A. Nielsen's contribution $^{22}$ ). But, on the other hand, issues like their nonuniqueness or the clairvoyant properties of trapped surfaces pose fundamental questions that cannot be ignored.

We do not aim here at addressing first-principles questions about the role of trapping horizons as a characterization of black hole horizons. We rather assume a pragmatic approach to the study of gravitational dynamics, which underlines the role of trapping horizons as hypersurfaces of remarkable geometric properties in black hole spacetimes. More specifically, our main interests are:

(i) The construction and diagnosis of black hole spacetimes in an Initial (Boundary) Value Problem approach.

(ii) Identification of a geometric probe into near-horizon spacetime dynamics.

Point (ii) is particularly important in the study of gravity in the strong-field regime, where the lack of rigid structures (e.g. symmetries, a background spacetime ... ) is 
a generic and essential problem. Given our interests and the adopted pragmatic methodology, we look for a geometric object such that: (a) represents a footprint of black holes, providing a probe into their geometry; (b) is adapted, by construction, to an Initial-Boundary Value Problem approach; and (c) although not-necessarily unique, provides a geometric structure with some sort of rigidity property. As we shall see in the following, dynamical trapping horizons fulfill these requirements.

\subsection{General scheme}

In Sec. 2 we introduce the basics of the geometry of closed surfaces in a Lorentzian manifold and motivate quasi-local horizons in stationary and dynamical regimes. Section 3 reviews their geometric properties and their special features as physical boundaries. Sections 4 and 5 are devoted to applications in a $3+1$ description of the spacetime. Section 4 shows the use of quasi-local horizons as inner boundary conditions for elliptic equations in General Relativity, whereas Sec. 5 discusses some applications to the analysis of spacetimes, in particular their role in a correlation approach to spacetime dynamics. In Sec. 6 a general overview is presented.

\section{Quasi-Local Horizons: Concepts and Definitions}

\subsection{Geometry of spacelike closed 2-surfaces $\mathcal{S}$}

\subsubsection{Normal plane: Outgoing and ingoing null vectors}

Let us consider a spacetime $\left(\mathcal{M}, g_{a b}\right)$ with Levi-Civita connection $\nabla_{a}$. Given a spacelike closed (compact without boundary) 2-surface $\mathcal{S}$ in $\mathcal{M}$ and a point $p \in \mathcal{S}$, the tangent space splits as $T_{p} \mathcal{M}=T_{p} \mathcal{S} \oplus T_{p}^{\perp} \mathcal{S}$. We span the normal plane $T_{p}^{\perp} \mathcal{S}$ either by (future-oriented) null vectors $\ell^{a}$ and $k^{a}$ (defined by the intersection between $T_{p}^{\perp} \mathcal{S}$ and the null cone at $p$ ) or by any pair of normal timelike vector $n^{a}$ and spacelike vector $s^{a}$. Let us denote conventionally $\ell^{a}$ to be the outgoing null normal and $k^{a}$ the ingoing one. We choose normalizations:

$$
\ell^{a} \ell_{a}=0, \quad k^{a} k_{a}=0, \quad \ell^{a} k_{a}=-1, \quad n^{a} n_{a}=-1, \quad s^{a} s_{a}=1, \quad n^{a} s_{a}=0 .
$$

Directions $\ell^{a}$ and $k^{a}$ are uniquely determined, but a normalization-boost freedom

$$
\begin{gathered}
\ell^{\prime a}=f \ell^{a}, \quad k^{\prime a}=f^{-1} k^{a}, \\
n^{\prime a}=\cosh (\sigma) n^{a}+\sinh (\sigma) s^{a}, \quad s^{\prime a}=\sinh (\sigma) n^{a}+\cosh (\sigma) s^{a},
\end{gathered}
$$

remains for some arbitrary rescaling positive function $f$ on $\mathcal{S}$ (where $\sigma=\ln (f)$ and $\ell^{a}=\lambda\left(n^{a}+s^{a}\right) / \sqrt{2}$ and $k^{a}=\lambda^{-1}\left(n^{a}-s^{a}\right) / \sqrt{2}$, for some function $\lambda$ on $\left.\mathcal{S}\right)$.

\subsubsection{Intrinsic geometry of $\mathcal{S}$}

The induced metric on $\mathcal{S}$ is given by

$$
q_{a b}=g_{a b}+k_{a} \ell_{b}+\ell_{a} k_{b}=g_{a b}+n_{a} n_{b}-s_{a} s_{b},
$$


so that $q^{a}{ }_{b}$ is the projector onto $\mathcal{S}$

$$
q_{b}^{a} q_{c}^{b}=q_{c}^{a}, \quad q_{b}^{a} v^{b}=v^{a}\left(\forall v^{a} \in T \mathcal{S}\right), \quad q_{b}^{a} w^{b}=0\left(\forall w^{a} \in T^{\perp} \mathcal{S}\right) .
$$

We denote the Levi-Civita connection associated with $q_{a b}$ as ${ }^{2} D_{a}$. The volume form on $\mathcal{S}$ will be denoted by ${ }^{2} \epsilon=\sqrt{q} d x^{1} \wedge d x^{2}$, i.e. ${ }^{2} \epsilon_{a b}=n^{c} s^{d 4} \epsilon_{c d a b}$, though we will also employ the area measure notation $d A=\sqrt{q} d^{2} x$.

\subsubsection{Extrinsic geometry of $\mathcal{S}$ in $(\mathcal{M}, g)$}

We define the second fundamental tensor of $\left(\mathcal{S}, q_{a b}\right)$ in $\left(\mathcal{M}, g_{a b}\right)$ (also, shape tensor or extrinsic curvature tensor) as

$$
\mathcal{K}_{a b}^{c}=q_{a}^{d} q_{b}^{e} \nabla_{d} q_{e}^{c},
$$

where $c$ is an index in the normal plane $T^{\perp} \mathcal{S}$, whereas $a$ and $b$ are indices in $T \mathcal{S}$. Given a vector $v^{a}$ normal to $\mathcal{S}$, we can define the deformation tensor $\Theta_{a b}^{(v)}$ as

$$
\Theta_{a b}^{(v)}=q_{a}^{c} q_{b}^{d} \nabla_{c} v_{d} .
$$

Then, using expression (4), the second fundamental tensor can be expressed as

$$
\mathcal{K}_{a b}^{c}=k^{c} \Theta_{a b}^{(\ell)}+\ell^{c} \Theta_{a b}^{(k)}=n^{c} \Theta_{a b}^{(n)}-s^{c} \Theta_{a b}^{(s)} .
$$

We can express $\Theta_{a b}^{(v)}$ in terms of the variation of the intrinsic metric along $v^{a}$. Given a (tensorial) object $A_{a_{1} \cdots a_{n}}{ }^{b_{1} \cdots b_{m}}$ tangent to $\mathcal{S}$ we denote by $\delta_{v}$ the operator $\left(\delta_{v} A\right)_{a_{1} \cdots a_{n}}{ }^{b_{1} \cdots b_{m}}=q_{a_{1}}{ }^{c_{1}} \cdots q_{a_{n}}{ }^{{ }^{n}}{ }^{2} q_{d_{1}}{ }^{b_{1}} \cdots q_{d_{m}}{ }^{b_{m}} \mathcal{L}_{v} A_{c_{1} \cdots c_{n}}{ }^{d_{1} \cdots d_{m}}$, where $\mathcal{L}_{v}$ denote the Lie derivative along (some extension of) $v^{a}$. Then, it follows

$$
\delta_{v} q_{a b}=\frac{1}{2} \Theta_{a b}^{(v)}
$$

(a) Shear and expansion associated with $v^{a}$. Defining the expansion $\theta^{(v)}$ and shear tensor $\sigma_{a b}^{(v)}$ associated with the normal vector $v^{a}$ as

$$
\theta^{(v)} \equiv q^{a b} \nabla_{a} v_{b}=\delta_{v} \ln \sqrt{q}, \quad \sigma_{a b}^{(v)} \equiv \Theta_{a b}^{(v)}-\frac{1}{2} \theta^{(v)} q_{a b},
$$

we express the deformation tensor $\Theta_{a b}^{(v)}$ in terms of his trace and traceless parts

$$
\Theta_{a b}^{(v)}=\sigma_{a b}^{(v)}+\frac{1}{2} \theta^{(v)} q_{a b}
$$

(b) Mean curvature vector $H^{a}$. Taking the trace of $\Theta_{a b}^{(v)}$ on $\mathcal{S}$ we define the mean curvature vector $^{\mathrm{a}}$

$$
H^{c} \equiv q^{a b} \mathcal{K}_{a b}^{c}=\theta^{(\ell)} k^{c}+\theta^{(k)} \ell^{c}
$$

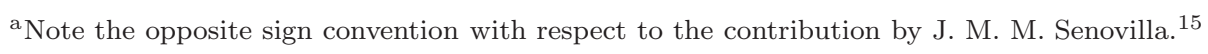


The extrinsic curvature information of $\left(\mathcal{S}, q_{a b}\right)$ in $\left(\mathcal{M}, g_{a b}\right)$ is completed by the normal fundamental forms associated with normal vectors $v^{a}$. In particular ${ }^{23}$

$$
\begin{aligned}
& \Omega_{a}^{(n)}=s^{c} q_{a}^{d} \nabla_{d} n_{c}, \quad \Omega_{a}^{(s)}=n^{c} q_{a}^{d} \nabla_{d} s_{c}, \\
& \Omega_{a}^{(\ell)}=\frac{1}{k^{b} \ell_{b}} k^{c} q_{a}^{d} \nabla_{d} \ell_{c}, \quad \Omega_{a}^{(k)}=\frac{1}{k^{b} \ell_{b}} \ell^{c} q^{d}{ }_{a} \nabla_{d} k_{c} .
\end{aligned}
$$

All these normal fundamental forms are related up to a sign and a total derivative on $\mathcal{S}$. Using the normalizations $(2)$ we get $^{\mathrm{b}}: \Omega_{a}^{(n)}=-\Omega_{a}^{(s)}, \Omega_{a}^{(\ell)}=-\Omega_{a}^{(k)}, \Omega_{a}^{(\ell)}=$ $\Omega_{a}^{(n)}-{ }^{2} D_{a} \lambda$. We choose to employ the 1 -form $\Omega_{a}^{(\ell)}$ in the following.

\subsubsection{Transformation properties under null normal rescaling}

Under the rescaling (2) $\ell^{a} \rightarrow f \ell^{a}, k^{a} \rightarrow f^{-1} k^{a}$ the introduced fields transform as

$$
\begin{array}{rlrl}
q_{a b} & \rightarrow q_{a b}, & { }^{2} D_{a} & \rightarrow{ }^{2} D_{a}, \\
\mathcal{K}_{a b}^{c} & \rightarrow \mathcal{K}_{a b}^{c}, & H^{a} & \rightarrow H^{a}, \\
\Theta_{a b}^{(\ell)} & \rightarrow f \Theta_{a b}^{(\ell)}, & \theta^{(\ell)} & \rightarrow f \theta^{(\ell)}, \\
\Theta_{a b}^{(k)} & \rightarrow f^{-1} \Theta_{a b}^{(k)}, & \theta^{(k)} & \rightarrow f^{-1} \theta^{(k)}, \quad \sigma_{a b}^{(\ell)} \rightarrow f \sigma_{a b}^{(\ell)}, \\
\Omega_{a}^{(\ell)} & \rightarrow \Omega_{a}^{(\ell)}+f^{-1} \sigma_{a b}^{(k)} .
\end{array}
$$

Finally, given an axial Killing vector $\phi^{a}$ on $\mathcal{S}$, we can write the angular momentum ${ }^{\mathrm{c}}$

$$
J=\frac{1}{8 \pi} \int_{\mathcal{S}} \Omega_{a}^{(\ell)} \phi^{a 2} \epsilon .
$$

The transformation rule of $\Omega_{a}^{(\ell)}$ in (14) together with the divergence-free property of $\phi^{a}$ (following from its Killing character) guarantee that the quantity $J$ does not depend on the choice of null normals $\ell^{a}, k^{a}$ (i.e. $J$ does not change under a boost).

\subsection{Trapping horizons}

\subsubsection{Worldtubes of marginally trapped surfaces}

A trapping horizon ${ }^{14}$ is (the closure of) a hypersurface $\mathcal{H}$ foliated by closed marginal (outer) trapped surfaces: $\mathcal{H}=\bigcup_{t \in \mathbb{R}} \mathcal{S}_{t}$, with $\left.\theta^{(\ell)}\right|_{\mathcal{S}_{t}}=0$. Trapping horizons are classified according to the signs of $\theta^{(k)}$ and $\delta_{k} \theta^{(\ell)}$. In particular, the sign of $\theta^{(k)}$ controls if the singularity occurs either in the future or in the past of $\mathcal{S}$, whereas the sign of $\delta_{k} \theta^{(\ell)}$ controls the (local) outer- or inner-most character of $\mathcal{H}$. Then, a trapping horizon is said to be: (i) future (respectively, past) if $\theta^{(k)}<0$ (respectively, $\theta^{(k)}>0$ ), and (ii) outer (respectively, inner) if there exists ${ }^{\mathrm{d}} \ell^{a}$ and $k^{a}$ such that $\delta_{k} \theta^{(\ell)}<0$ (respectively, $\delta_{k} \theta^{(\ell)}>0$ ).

${ }^{\mathrm{b}}$ When using $\ell^{a} k_{a}=-e^{\sigma}$ one gets: $\Omega_{a}^{(\ell)}=-\Omega_{a}^{(k)}-{ }^{2} D_{a} \sigma$. This will be relevant later, in Eq. (39). ${ }^{\mathrm{c}}$ The quantity $J$ coincides with the Komar angular momentum in case that $\phi^{a}$ can be extended to an axial Killing in the neighborhood of $\mathcal{S}$.

d The sign of $\delta_{k} \theta^{(\ell)}$ is not invariant on the whole $\mathcal{S}$ under a rescaling (2). However, if there exists $\ell^{a}$ and $k^{a}$ such that $\delta_{k} \theta^{(\ell)}<0$ on $\mathcal{S}$, then there does not exist any choice of $\ell^{a}$ and $k^{a}$ such that $\delta_{k} \theta^{(\ell)}>0$ on $\mathcal{S}$; see Ref. 24 and also the marginally trapped surface stability condition in Ref. 25 . 


\subsubsection{Future outer trapping horizons}

In a black hole setting the singularity occurs in the future of sections $\mathcal{S}_{t}$ of $\mathcal{H}$, so that the related trapping horizon is of future type, $\theta^{(k)}<0$. In addition, when considering displacements along $k^{a}$ (ingoing direction) we should move into the trapped region, i.e. $\delta_{k} \theta^{(\ell)}<0$, so that the trapping horizon should be outer.

The resulting characterization of quasi-local black hole horizons as Future Outer Trapping Horizons (FOTHs) is further supported by the following analysis of the area evolution. Hawking's area theorem for event horizons (cf. Sec. 1.2) captures a fundamental feature of classical black holes. It is natural to wonder about a quasilocal version of it. Let us consider an evolution vector $h^{a}$ along the trapping horizon $\mathcal{H}$, characterized as: (i) $h^{a}$ is tangent to $\mathcal{H}$ and orthogonal to $\mathcal{S}_{t}$, and (ii) $h^{a}$ transports $\mathcal{S}_{t}$ onto $\mathcal{S}_{t+\delta t}: \delta_{h} t=1$. We can write $h^{a}$ and a dual vector $\tau^{a}$ orthogonal to $\mathcal{H}$ as

$$
h^{a}=\ell^{a}-C k^{a}, \quad \tau^{a}=\ell^{a}+C k^{a} .
$$

Then $h^{a} h_{a}=-\tau^{a} \tau_{a}=2 C$, i.e. $h^{a}$ is spacelike for $C>0$, null for $C=0$ and timelike for $C<0$. The evolution of the area $A=\int_{\mathcal{S}} d A=\int_{\mathcal{S}}{ }^{2} \epsilon$ along $h^{a}$ is given by

$$
\delta_{h} A=\int_{\mathcal{S}} \theta^{(h) 2} \epsilon=\int_{\mathcal{S}}\left(\theta^{(\ell)}-C \theta^{(k)}\right)^{2} \epsilon=-\int_{\mathcal{S}} C \theta^{(k) 2} \epsilon .
$$

Considering for simplicity the spherical symmetric case $(C=$ const; see discussion of Eq. (37) in 3.2.4, for the general case), the trapping horizon condition, $\delta_{h} \theta^{(\ell)}=0$, writes $\delta_{\ell} \theta^{(\ell)}-C \delta_{k} \theta^{(\ell)}=0$, so that $C=\frac{\delta_{\ell} \theta^{(\ell)}}{\delta_{k} \theta^{(\ell)}}$. Applying the Raychaudhuri equation for $\delta_{\ell} \theta^{(\ell)}$ [see later Eq. (21)], together with the $\theta^{(\ell)}=0$ condition, we find

$$
C=-\frac{\sigma_{a b}^{(\ell)} \sigma^{(\ell)}{ }^{a b}+8 \pi T_{a b} \ell^{a} \ell^{b}}{\delta_{k} \theta^{(\ell)}} .
$$

Under the null energy and outer horizon conditions, it follows $C \geq 0$, so that the future condition guarantees the nondecrease of the area in (17). Therefore, FOTHs are null or spacelike hypersurfaces $(C \geq 0)$, satisfying an area law result, and therefore providing appropriate models for quasi-local black hole horizons.

\subsection{Isolated and dynamical horizons}

The distinct geometric structure of null and spatial hypersurfaces suggests different strategies for the study of the stationary and dynamical regimes of quasi-local black holes, modeled as future outer trapping horizons. This has led to the parallel development of the isolated horizon and the dynamical horizon frameworks. ${ }^{26-29}$

In equilibrium, Isolated Horizons (IH) provide a hierarchy of geometric structures constructed on a null hypersurface $\mathcal{H}$ that is foliated by closed (outer) marginally trapped surfaces. They characterize different levels of stationarity for a black hole horizon in an otherwise dynamical environment:

(i) Non-Expanding Horizons (NEH). They represent the minimal notion of equilibrium by imposing the stationarity of the intrinsic geometry $q_{a b}$. 
(ii) Weakly Isolated Horizons (WIH). They are NEHs endowed with an additional structure needed for a Hamiltonian analysis of the horizon and its related (thermo-)dynamics. They impose no additional constraints on the geometry of the NEH.

(iii) Isolated Horizons (IH). These are WIHs whose extrinsic geometry is also invariant along the evolution. They provide the strongest stationarity notion on $\mathcal{H}$.

The nonstationary regime can be characterized by Dynamical Horizons (DH), namely spacelike hypersurfaces $\mathcal{H}$ foliated by closed future marginally trapped surfaces, i.e. $\theta^{(\ell)}=0$ and $\theta^{(k)}<0$. Introduced in a $3+1$ formulation, they provide a complementary perspective to the dual-null foliation formulation ${ }^{14}$ of trapping horizons, making them naturally adapted for an Initial Value Problem perspective.

\subsection{IHs and DHs as stationary and dynamical sections of FOTHs}

A natural question when considering the transition from equilibrium to the dynamical regime is whether a section $\mathcal{S}_{t}$ of a FOTH can be partially stationary and partially dynamical. Or, in other words, whether the element of area $d A$ can be nonexpanding $(C=0)$ in a part of $\mathcal{S}_{t}$ whereas it already expands $(C>0)$ in another part. Namely, can $h^{a}$ be both null and spacelike on a section $\mathcal{S}_{t}$ of a FOTH?

The answer is in the negative. Transitions between non-expanding and dynamical parts of a FOTH must happen all at once. More precisely, assuming the null energy condition, a FOTH can be completely partitioned into non-expanding and dynamical sections. For a section $\mathcal{S}_{t}$ to be completely dynamical $(C>0)$ it suffices that it has $\delta_{\ell} \theta^{(\ell)}<0$ somewhere on it. Otherwise $h^{a}$ is null $(C=0)$ all over $\mathcal{S}_{t}{ }^{24,30}$

In more physical terms, it suffices that some energy crosses the horizon somewhere, and the whole horizon instantaneously grows as a whole. This nonlocal behavior is a consequence of the elliptic nature of quasi-local horizons. As shown in Sec. 3.2.3, the function $C$ determining the metric type of $h^{a}$ satisfies an elliptic equation [cf. Eq. (37)]. Under the outer condition $\delta_{k} \theta^{(\ell)}<0$ one can apply a maximum principle to show that $C$ is non-negative [generalization of Eq. (18)]. Moreover, it suffices that $\delta_{\ell} \theta^{(\ell)} \neq 0$ somewhere, for having $C>0$ everywhere.

\section{Quasi-Local Horizons: Properties from a $3+1$ Perspective}

\subsection{Equilibrium regime}

\subsubsection{Null hypersurfaces: Characterization and basic elements}

A hypersurface $\mathcal{H}$ is null if and only if the induced metric is degenerated. Equivalently, if and only if there is a tangent null vector $\ell^{a}$ orthogonal to all vectors tangent to $\mathcal{H}: \ell^{a} v_{a}=0, \forall v^{a} \in T \mathcal{H}$.

Let us introduce some elements on the geometry of $\mathcal{H}$. Choosing a null vector $k^{a}$ transverse to $\mathcal{H}$, we can write $\mathrm{e}^{\mathrm{e}}$ the degenerated metric as $q_{a b}=g_{a b}+k_{a} \ell_{b}+\ell_{a} k_{b}$.

${ }^{\text {e}}$ We abuse notation and employ the same notation employed in sections $\mathcal{S}_{t}$ of $\mathcal{H}$, cf. Eq. (4). 
A projector onto $\mathcal{H}$ can also be constructed as: $\Pi_{a}{ }^{b}=\delta_{a}{ }^{b}+\ell_{a} k^{b}=q_{a}{ }^{b}-k_{a} \ell^{b}$. As a part of the extrinsic curvature of $\mathcal{H}$, a rotation 1 -form can be introduced ${ }^{31}$ on $\mathcal{H}$ as $\omega_{a}^{(\ell)}=\frac{1}{\ell^{a} k_{a}} k^{c} \nabla_{a} \ell_{c}$. This 1 -form lives on $\mathcal{H}$, i.e. $k^{a} \omega_{a}^{(\ell)}=0$. In particular, we can write $\Pi_{a}{ }^{c} \nabla_{c} \ell^{b}=\omega_{a}^{(\ell)} \ell^{b}+\Theta^{(\ell)}{ }_{a}^{b}$, where $\Theta_{a b}^{(\ell)}$ is given by expression (7) [cf. Eq. (5.23) in Ref. 28]. Contracting with $\ell^{a}$ we find: $\ell^{c} \nabla_{c} \ell^{a}=\kappa^{(\ell)} \ell^{a}$, a pre-geodesic equation where the non-affinity coefficient $\kappa^{(\ell)}$ is defined as $\kappa^{(\ell)}=\ell^{a} \omega_{a}^{(\ell)}$. If a foliation $\left\{\mathcal{S}_{t}\right\}$ of $\mathcal{H}$ is given, we can write [cf. Eq. (5.35) in Ref. 28]: $\omega_{a}^{(\ell)}=\Omega_{a}^{(\ell)}-\kappa^{(\ell)} k_{a}$.

Vectors $\ell^{a}$ and $k^{a}$ can be completed to a tetrad $\left\{\ell^{a}, k^{a},\left(e_{1}\right)^{a},\left(e_{2}\right)^{a}\right\}$, where $\left(e_{i}\right)^{a}$ are tangent to sections $\mathcal{S}_{t}$. Normalizations given in (2) are then completed to

$$
\ell \cdot\left(e_{i}\right)_{a}=0, \quad k^{a}\left(e_{i}\right)_{a}=0, \quad\left(e_{i}\right)^{a}\left(e_{i}\right)_{b}=\delta_{a b} .
$$

Defining the complex null vector $m^{a}=\frac{1}{\sqrt{2}}\left[\left(e_{1}\right)^{a}+i\left(e_{2}\right)^{a}\right]$, the Weyl scalars are defined as the components of the Weyl tensor $C^{a}{ }_{b c d}$ in the null tetrad $\left\{\ell^{a}, k^{a}, m^{a}, \bar{m}^{a}\right\}$

$$
\begin{array}{ll}
\Psi_{0}=C^{a}{ }_{b c d} \ell_{a} m^{b} \ell^{c} m^{d}, & \Psi_{3}=C_{b c d}^{a} \ell_{a} k^{b} \bar{m}^{c} k^{d}, \\
\Psi_{1}=C^{a}{ }_{b c d} \ell_{a} m^{b} \ell^{c} k^{d}, & \Psi_{4}=C^{a}{ }_{b c d} \bar{m}_{a} k^{b} \bar{m}^{c} k^{d} . \\
\Psi_{2} & =C^{a}{ }_{b c d} \ell_{a} m^{b} \bar{m}^{c} k^{d},
\end{array}
$$

\subsubsection{Null hypersurfaces: Evolution}

It is illustrative to give a $3+1$ perspective on $\mathcal{H}$. Given a foliation $\mathcal{H}=\bigcup_{t \in \mathbb{R}} \mathcal{S}_{t}$ let us evaluate explicitly the evolution along $\ell^{a}$ of some quantities defined on sections $\mathcal{S}_{t}$.

(i) Expansion equation (null Raychaudhuri equation):

$$
\delta_{\ell} \theta^{(\ell)}-\kappa^{(\ell)} \theta^{(\ell)}+\frac{1}{2} \theta^{(\ell)^{2}}+\sigma_{a b}^{(\ell)} \sigma^{(\ell)^{a b}}+8 \pi T_{a b} \ell^{a} \ell^{b}=0 .
$$

(ii) Tidal equation:

$$
\delta_{\ell} \sigma_{a b}^{(\ell)}=\kappa^{(\ell)} \sigma_{a b}^{(\ell)}+\sigma_{c d}^{(\ell)} \sigma^{(\ell)}{ }^{c d} q_{a b}-q_{a}^{c} q_{b}^{d} C_{e c f d} \ell^{e} \ell^{f}
$$

(iii) Evolution for $\Omega_{a}$ :

$$
\delta_{\ell} \Omega_{c}^{(\ell)}+\theta^{(\ell)} \Omega_{a}^{(\ell)}=8 \pi T_{c d} \ell^{c} q_{a}^{d}+{ }^{2} D_{a}\left(\kappa^{(\ell)}+\frac{\theta^{(\ell)}}{2}\right)-{ }^{2} D_{c} \sigma^{(\ell)^{c}}{ }_{a} .
$$

\subsubsection{Non-expanding horizons}

A $\mathrm{NEH}^{32}$ is a null-hypersurface $\mathcal{H} \approx S^{2} \times \mathbb{R}$, on which the expansion associated with $\ell^{a}$ vanishes $\left(\theta^{(\ell)}=0\right)$, the Einstein equations hold and $-T_{c}^{a} \ell^{c}$ is future directed (null dominant energy condition). Note that any foliation $\mathcal{H}=\bigcup_{t \in \mathbb{R}} \mathcal{S}_{t}$ produces a foliation of $\mathcal{H}$ by MOTS $\mathcal{S}_{t}$.

(i) NEH characterization. Making $\theta^{(\ell)}=0$ in the Raychaudhuri Eq. (21) we get

$$
\sigma_{a b} \sigma^{a b}+8 \pi T_{a b} \ell^{a} \ell^{b}=0 .
$$


Since the two terms are positive-definite, they vanish independently. This provides an instantaneous characterization of a NEH:

$$
\theta^{(\ell)}=0, \quad \sigma_{a b}^{(\ell)}=0, \quad T_{a b} \ell^{a} \ell^{b}=0 .
$$

From Eq. (11) with $v^{a}=\ell^{a}$, it follows $\Theta_{a b}^{(\ell)}=0$. The NEH characterization is equivalent, cf. Eq. (9), to the evolution independence of the induced metric $q_{a b}$

$$
\delta_{\ell} q_{a b}=\frac{1}{2} \Theta_{a b}^{(\ell)}=0 .
$$

From Eq. (8), we conclude that a NEH fixes half of the degrees of freedom in the second fundamental form $\mathcal{K}_{a b}^{c}$ of $\mathcal{S}_{t}$ in $\mathcal{M}$. This will be relevant in Sec. 4.2.1.

(ii) Connection $\hat{\nabla}_{a}$ on a NEH. A null hypersurface has no unique (Levi-Civita) connection compatible with the metric. However, on a NEH $\mathcal{H}$ one can introduce a preferred connection as that one induced from the spacetime connection $\nabla_{a}: u^{c} \hat{\nabla}_{c} w^{a} \equiv u^{c} \nabla_{c} w^{a}, \forall u^{a}, w^{a} \in T \mathcal{H}$. Indeed using NEH characterization (26), $u^{c} \nabla_{c} w^{a}$ is tangent to $\mathcal{H}: \ell_{d}\left(u^{c} \nabla_{c} w^{d}\right)=u^{c} \nabla_{c}\left(\ell_{d} w^{d}\right)-u^{c} w^{d} \Theta_{c d}^{(\ell)}=0$.

(iii) Geometry of a NEH. We refer (cf. Ref. 33) to the pair $\left(q_{a b}, \hat{\nabla}_{a}\right)$ as the geometry of a NEH. Writing the components of the $\hat{\nabla}_{a}$ connection in terms of quantities on $\mathcal{S}_{t}$

$$
\begin{aligned}
q_{a}^{c}{ }_{a} q_{d}^{b} \hat{\nabla}_{c} v^{d} & ={ }^{2} D_{a}\left(q^{b}{ }_{c} v^{c}\right), \\
q^{c}{ }_{a} k_{d} \hat{\nabla}_{c} v^{d} & ={ }^{2} D_{a}\left(v^{c} k_{c}\right)-q_{a}^{c} v^{d} \Theta_{c d}^{(k)}, \\
\ell^{c} \hat{\nabla}_{c} v^{a} & =\delta_{\ell} v^{a}+v^{c} \omega_{c}^{(\ell)} \ell^{a},
\end{aligned}
$$

the free data on a NEH are given, from an evolution perspective, by $\left(\left.q_{a b}\right|_{\mathcal{S}_{t}},\left.\Omega_{a}^{(\ell)}\right|_{\mathcal{S}_{t}},\left.\kappa^{(\ell)}\right|_{\mathcal{H}},\left.\Theta_{a b}^{(k)}\right|_{\mathcal{S}_{t}}\right)$, where $q_{a b}$ is time independent.

(iv) Weyl tensor on a NEH. Under the rescaling (3), the 1 -form $\omega_{a}^{(\ell)}$ transforms as $\omega_{a}^{(\ell)} \rightarrow \omega_{a}^{(\ell)}+\hat{\nabla}_{a} \ln f$. Its exterior derivative $d \omega^{(\ell)}$ provides a gauge invariant object: understanding $\omega_{a}^{(\ell)}$ as a gauge connection, $d \omega^{(\ell)}$ is its gauge-invariant curvature. Using the NEH condition, $\Theta_{a b}^{(\ell)}=0$, one can express (cf. Sec. 7.6.2. in Ref. 28)

$$
d \omega^{(\ell)}=2 \operatorname{Im} \Psi_{2}^{2} \epsilon
$$

Hence, $\operatorname{Im} \Psi_{2}$ is gauge invariant on a NEH. Actually the full $\Psi_{2}$ is invariant, as it follows from its boost transformation rules and the values of $\Psi_{0}$ and $\Psi_{1}$ on a $\mathrm{NEH},{ }^{28}$

$$
\left.\Psi_{0}\right|_{\mathcal{H}}=\left.\Psi_{1}\right|_{\mathcal{H}}=0
$$

\subsubsection{Weakly isolated horizons}

A Weakly Isolated Horizon $(\mathrm{WIH})\left(\mathcal{H},\left[\ell^{a}\right]\right)$ is a NEH together with a class of null normals $\left[\ell^{a}\right]$ such that: $\delta_{\ell} \omega_{a}^{(\ell)}=0$. This condition permits to set a well-posed variational problem for spacetimes containing stationary quasi-local horizons. This 
enables the development of a Hamiltonian analysis on the horizon $\mathcal{H}$ leading to the construction of conserved quantities under WIH-symmetries. ${ }^{31}$ In particular, the expression for the angular momentum in Eq. (15) is recovered

$$
J_{\mathcal{H}}=\frac{1}{8 \pi} \int_{\mathcal{S}_{t}} \omega_{c}^{(\ell)} \phi^{c 2} \epsilon=\frac{1}{8 \pi} \int_{\mathcal{S}_{t}} \Omega_{c}^{(\ell)} \phi^{c 2} \epsilon=-\frac{1}{4 \pi} \int_{\mathcal{S}_{t}} f \operatorname{Im} \Psi_{2}{ }^{2} \epsilon,
$$

with $\phi^{a}={ }^{2} D_{c} f^{2} \epsilon^{a c}$ ( $\phi^{a}$ is an axial Killing vector, in particular divergence-free).

The WIH structure is relevant for the discussion of IH thermodynamics (cf. A. Nielsen's contribution ${ }^{22}$ ). We do not address this issue here and just comment on the equivalence of the WIH condition with a thermodynamical zeroth law. Reminding $\omega_{a}^{(\ell)}=\Omega_{a}^{(\ell)}-\kappa^{(\ell)} k_{a}$, the (vacuum) evolution equation $(23)$ for $\Omega_{a}^{(\ell)}$ leads to $\mathcal{L}_{\ell} \Omega_{a}^{(\ell)}=$ ${ }^{2} D_{a} \kappa^{(\ell)}$. More generally, $\delta_{\ell} \omega_{a}^{(\ell)}=\hat{\nabla} \kappa^{(\ell)}$ (cf. for example Eq. (8.5) in Ref. 28). That is, on WIHs the non-affinity coefficient (surface gravity) is constant: $\kappa^{(\ell)}=\kappa_{o}$.

WIHs and NEH geometry. WIHs do not constraint the underlying NEH geometry. In other words, every NEH admits a WIH structure. In fact, given $\kappa^{(\ell)} \neq$ const., the rescaling $\ell^{\prime}=\alpha \ell$, with $\kappa_{o}=$ const. $=\nabla_{\ell} \alpha+\alpha \kappa^{(\ell)}$, leads to a constant $\kappa^{\left(\ell^{\prime}\right)}=\kappa_{O}$. Finally, free data for a WIH are again $\left(q_{a b}\left|{ }_{\mathcal{S}_{t}}, \Omega_{a}^{(\ell)}\right| \mathcal{S}_{t},\left.\kappa^{(\ell)}\right|_{\mathcal{H}},\left.\Theta_{a b}^{(k)}\right|_{\mathcal{S}_{t}}\right)$, but now $q_{a b} \mid \mathcal{S}_{t}$, $\left.\Omega_{a}^{(\ell)}\right|_{\mathcal{S}_{t}}$ and $\left.\kappa^{(\ell)}\right|_{\mathcal{H}}=\kappa_{o}$ are time-independent.

\subsection{5. (Strongly) isolated horizons}

An isolated horizon (IH) is a WIH on which the whole extrinsic geometry is timeinvariant: $\left[\delta_{\ell}, \hat{\nabla}_{a}\right]=0$. This condition can be characterized ${ }^{28,33}$ as $\delta_{\ell} \Theta^{(k)}=0$, that leads to the geometric constraint

$$
\kappa^{(\ell)} \Theta_{a b}^{(k)}=\frac{1}{2}\left({ }^{2} D_{a} \Omega_{b}^{(\ell)}+{ }^{2} D_{b} \Omega_{a}^{(\ell)}\right)+\Omega_{a}^{(\ell)} \Omega_{b}^{(\ell)}-\frac{1}{2}{ }^{2} R_{a b}+4 \pi\left(q_{a}^{c} q_{b}^{d} T_{c d}-\frac{T}{2} q_{a b}\right) .
$$

With Eq. (26), this fixes completely the second fundamental form $\mathcal{K}_{a b}^{c}$. Free data of an IH, $\left(q_{a b}\left|\mathcal{S}_{t}, \Omega_{a}^{(\ell)}\right|_{\mathcal{S}_{t}},\left.\kappa^{(\ell)}\right|_{\mathcal{H}}=\kappa_{o}\right)$, are time independent. Their geometric (gaugeinvariant) content can be encoded in the pair ${ }^{\mathrm{f}}:\left({ }^{2} R, \operatorname{Im} \Psi_{2}\right)$. On the one hand, ${ }^{2} R$ accounts for the gauge-invariant part of $q_{a b}$. Regarding $\Omega_{a}^{(\ell)}$, from $d \omega^{(\ell)}=2 \operatorname{Im} \Psi_{2}^{2} \epsilon$ and $\kappa^{(\ell)}=$ const., it follows $d \Omega^{(\ell)}=2 \operatorname{Im} \Psi_{2}^{2} \epsilon$. On a sphere $\mathcal{S}_{t}$ we can write $\Omega_{a}^{(\ell)}=\Omega_{a}^{\text {div-free }}+\Omega_{a}^{\text {exact }}$, so that $\Omega_{a}^{\text {exact }}={ }^{2} D_{a} g$ is gauge-dependent [cf. (14)]. From $d \Omega_{a}^{\text {div-free }}=2 \operatorname{Im} \Psi_{2}$, the gauge-invariant part of $\Omega_{a}^{(\ell)}$ is encoded $\operatorname{in} \operatorname{Im} \Psi_{2}$.

IH multipoles of axially symmetric horizons. On an axially symmetric IH, the gaugeinvariant part of the geometry, $\left({ }^{2} R, \operatorname{Im} \Psi_{2}\right)$, can be decomposed onto spherical harmonics. On an axially symmetric section $\mathcal{S}_{t}$ of $\mathcal{H}$, a coordinate system can be

${ }^{\mathrm{f}}$ Note the relation with the complex scalar $\mathcal{K}$ in Refs. 34 and 35. 
canonically constructed, ${ }^{36,37}$ such that $\left[\right.$ with $\left.A_{\mathcal{H}}=4 \pi\left(R_{\mathcal{H}}\right)^{2}\right]$

$$
q_{a b} d x^{a} \otimes d x^{b}=\left(R_{\mathcal{H}}\right)^{2}\left(F^{-1} \sin ^{2} \theta d \theta \otimes d \theta+F d \phi \otimes d \phi\right) .
$$

In particular, $d A=\left(R_{\mathcal{H}}\right)^{2} \sin \theta d \theta d \phi$ (round sphere area element). We can then use standard spherical harmonics $Y_{\ell m}(\theta)$, with $m=0$ in this axisymmetric case

$$
\int_{\mathcal{S}_{t}} Y_{\ell 0}(\theta) Y_{\ell^{\prime} 0}(\theta) d^{2} A=\left(R_{\mathcal{H}}\right)^{2} \delta_{\ell \ell^{\prime}},
$$

to define the $I H$ geometric multipoles ${ }^{36} I_{n}$ and $L_{n}$

$$
\begin{aligned}
I_{n} & =\frac{1}{4} \int_{\mathcal{S}_{t}} R Y_{n 0}(\theta) d^{2} A, \\
L_{n} & =-\int_{\mathcal{S}_{t}} \operatorname{Im} \Psi_{2} Y_{n 0}(\theta) d^{2} A .
\end{aligned}
$$

Then, mass $M_{n}$ and angular momentum $J_{n}$ multipoles are defined ${ }^{36-39}$ by adequate dimensional rescalings of $I_{n}$ and $L_{n}$.

\subsubsection{Gauge freedom on a NEH: Non-uniqueness of the foliation}

Before proceeding to the dynamical case, we underline the existence of a fundamental gauge freedom in the equilibrium (null) case: any foliation $\left\{\mathcal{S}_{t}\right\}$ of a $N E H$ $\mathcal{H}$ provides a foliation of $\mathcal{H}$ by marginally trapped surfaces. This is equivalent to the rescaling freedom of the null normal $\ell^{a} \rightarrow f \ell^{a}$. Therefore, the amount of gauge freedom in the equilibrium case is encoded in one arbitrary function $f$ on $\mathcal{S}_{t}$.

Note that in this equilibrium horizon context, the relevant spacetime geometric object (the hypersurface $\mathcal{H}$ ) is unique, whereas the gauge-freedom enters in its evolution description due to the non-uniqueness of its possible foliation by MOTS.

\subsection{Dynamical case}

\subsubsection{Existence and foliation uniqueness results}

Let us introduce two fundamental results following from the application of geometric analysis techniques to the study of dynamical trapping horizons.

Property 1 (Dynamical horizon foliation uniqueness). ${ }^{40}$ Given a dynamical FOTH $\mathcal{H}$, the foliation by marginally trapped surfaces is unique.

This first result identifies an important rigidity property of DHs: the uniqueness of its evolution description. This is in contrast with the equilibrium null case, with its freedom in the choice of the foliation. In particular, on a dynamical FOTH the evolution vector is completely determined: $h^{a}$ is unique up to time reparametrization.

Property 2 (Existence of DHs). ${ }^{\mathbf{3 0}, 41}$ Given a marginally trapped surface $\mathcal{S}_{0}$ satisfying an appropriate stability condition on a Cauchy hypersurface $\Sigma$, to each $3+1$ 
spacetime foliation $\left(\Sigma_{t}\right)_{t \in \mathbb{R}}$ it corresponds a unique dynamical FOTHs $\mathcal{H}$ containing $\mathcal{S}_{0}$ and sliced by marginally trapped surfaces $\left\{\mathcal{S}_{t}\right\}$ such that $\mathcal{S}_{t} \subset \Sigma_{t}$.

This second result addresses the Initial Value Problem of DHs, in particular the existence of an evolution for a given MOTS into a dynamical FOTH. The result requires a stability condition (namely, $\mathcal{S}_{0}$ is required to be stably outermost $\left.{ }^{25,30,41,42}\right)$, so that the sign of the variation of $\theta^{(\ell)}$ in the inward (outward) direction is under control. This is essentially the outer condition ${ }^{14}$ in the FOTH characterization.

\subsection{2. "Gauge" freedom: Non-uniqueness of dynamical horizons}

The evolution of an $\mathrm{AH}$ into a $\mathrm{DH}$ is non-unique, as a consequence of combining Properties 1 and 2 above. Let us consider an initial AH $\mathcal{S}_{0} \subset \Sigma_{0}$ and two different $3+1$ slicings $\left\{\Sigma_{t_{1}}\right\}$ and $\left\{\Sigma_{t_{2}}\right\}$, compatible with $\Sigma_{0}$. From Property 2 there exist DHs $\mathcal{H}_{1}=\bigcup_{t_{1}} \mathcal{S}_{t_{1}}$ and $\mathcal{H}_{2}=\bigcup_{t_{2}} \mathcal{S}_{t_{2}}$, with $\mathcal{S}_{t_{1}}=\mathcal{H}_{1} \cap \Sigma_{t_{1}}$ and $\mathcal{S}_{t_{2}}=\mathcal{H}_{2} \cap \Sigma_{t_{2}}$ marginally trapped surfaces. Let us consider now the sections of $\mathcal{H}_{1}$ by $\left\{\Sigma_{t_{2}}\right\}$, i.e. $\mathcal{S}_{t_{2}}^{\prime}=\mathcal{H}_{1} \cap \Sigma_{t_{2}}$, so that $\mathcal{H}_{1}=\bigcup_{t_{2}} \mathcal{S}_{t_{2}}^{\prime}$. In the generic case, slicings $\left\{\mathcal{S}_{t_{2}}^{\prime}\right\}$ and $\left\{\mathcal{S}_{t_{1}}\right\}$ of $\mathcal{H}_{1}$ are different (one can consider a deformation of the slicing $\left\{\Sigma_{t_{2}}\right\}$, if needed). Therefore, from the foliation uniqueness of Property 1 , sections $\mathcal{S}_{t_{2}}^{\prime}$ cannot be marginally trapped surfaces. It follows then that $\mathcal{H}_{1}$ and $\mathcal{H}_{2}$ are different as hypersurfaces in $\mathcal{M}$ : if $\mathcal{H}_{1}=\mathcal{H}_{2}$, sections $\mathcal{S}_{t_{2}}$ (MOTSs) and $\mathcal{S}_{t_{2}}^{\prime}$ (non-MOTSs) would coincide by construction, leading to a contradiction. In addition to this non-uniqueness, DHs interweave in spacetime due to the existence of causal constraints ${ }^{40}$ : a $\mathrm{DH} \mathcal{H}_{1}$ cannot lay completely in the causal past of another $\mathrm{DH} \mathcal{H}_{2}$ (cf. Fig. 1).

Comparing with the discussion in Sec. 3.1.6 on the uniqueness and gaugefreedom issues in the equilibrium case, we conclude from the previous geometric

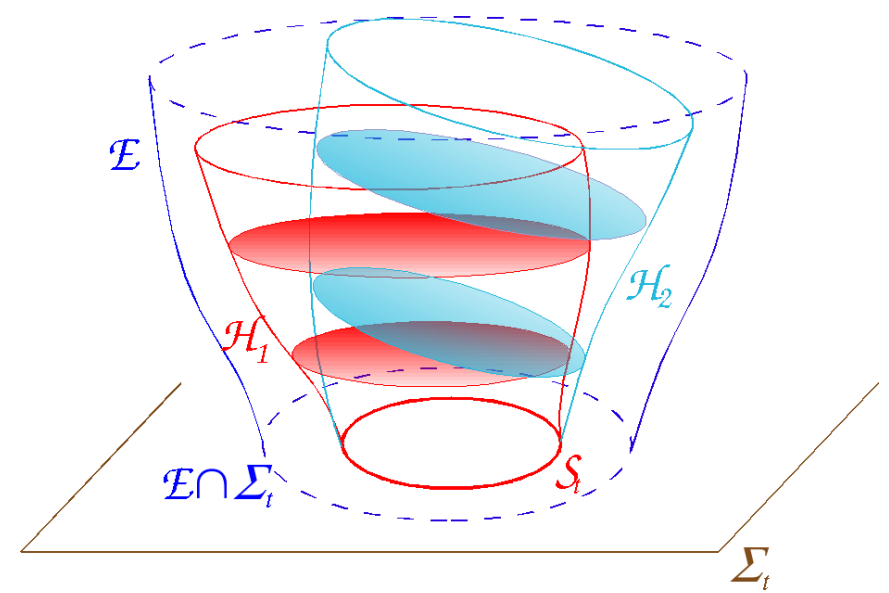

Fig. 1. (Color online) Illustration of the DH non-uniqueness. Dynamical horizons $\mathcal{H}_{1}$ and $\mathcal{H}_{2}$ represent evolutions from a given initial MOTS corresponding to different spacetime $3+1$ slicings. 
considerations that the dynamical and equilibrium cases contain the same amount of gauge freedom, namely a function on $\mathcal{S}$, although dressed in a different form. More specifically, whereas in the NEH case there is a fixed horizon, with a rescaling freedom ( $\ell^{a} \rightarrow f \ell^{a}, f$ function on $\mathcal{S}_{t}$ ), in the $\mathrm{DH}$ case the foliation is fixed, but a (gauge) freedom appears in the choice of the evolving horizon (lapse function $N$ on $\left.\mathcal{S}_{t}\right)$. In other words, in the dynamical case the choice is among distinct spacetime geometric objects, $\mathcal{H}_{1}$ and $\mathcal{H}_{2}$, whereas in the equilibrium case the choice concerns the description (foliation) of a single spacetime geometric object $\mathcal{H}$.

\subsubsection{FOTH characterization}

As discussed in Subsec. 2.2, a FOTH with evolution vector $h^{a}=\ell^{a}-C k^{a}$ is characterized by: (i) a trapping horizon condition: $\theta^{(\ell)}=0, \delta_{h} \theta^{(\ell)}=0$, (ii) a future condition $\theta^{(k)}<0$, and (iii) an outer condition: $\delta_{k} \theta^{(\ell)}<0$. These conditions can be made more explicit in terms of the variations ${ }^{24,43}$

$$
\begin{aligned}
& \delta_{\alpha \ell} \theta^{(\ell)}=-\alpha\left(\sigma_{a b}^{(\ell)} \sigma^{(\ell)^{a b}}-8 \pi T_{a b} \ell^{a} \ell^{b}\right), \\
& \delta_{\beta k} \theta^{(\ell)}=\beta\left[-{ }^{2} D^{c} \Omega_{c}^{(\ell)}+\Omega_{c}^{(\ell)} \Omega^{(\ell)^{c}}-\frac{1}{2}{ }^{2} R+8 \pi T_{a b} k^{a} \ell^{b}\right]+{ }^{2} \Delta \beta-2 \Omega_{c}^{(\ell) 2} D^{c} \beta,
\end{aligned}
$$

with $\alpha$ and $\beta$ functions on $\mathcal{S}_{t}$. Making $\beta=1$, the outer condition writes

$$
\delta_{k} \theta^{(\ell)}=-{ }^{2} D^{c} \Omega_{c}^{(\ell)}+\Omega_{c}^{(\ell)} \Omega^{(\ell)^{c}}-\frac{1}{2}^{2} R+8 \pi T_{a b} k^{a} \ell^{b}<0,
$$

for some $\ell^{a}$ and $k^{a}$, whereas the trapping horizon condition (with $\alpha=1, \beta=C$ ) is

$$
\delta_{h} \theta^{(\ell)}=\delta_{\ell} \theta^{(\ell)}-\delta_{C k} \theta^{(\ell)}=\delta_{\ell} \theta^{(\ell)}-C \delta_{k} \theta^{(\ell)}-{ }^{2} \Delta C+2 \Omega_{c}^{(\ell) 2} D^{c} C=0,
$$

that is

$$
-{ }^{2} \Delta C+2 \Omega_{c}^{(\ell) 2} D^{c} C-C\left[-{ }^{2} D^{c} \Omega_{c}^{(\ell)}+\Omega_{c}^{(\ell)} \Omega^{(\ell)^{c}}-\frac{1}{2} 2 R\right]=\sigma_{a b}^{(\ell)} \sigma^{(\ell)}{ }^{a b}+8 \pi T_{a b} \tau^{a} \ell^{b}
$$

This elliptic condition on $C$, in particular through the application of a maximum principle relying on the outer condition $\delta_{k} \theta^{(\ell)}<0$, is at the heart of the nonlocal behavior of the worldtube $\bigcup_{t \in \mathbb{R}} \mathcal{S}_{t}$ discussed in Sec. 2.4.

Remark on the variation/deformation/stability operator $\delta_{v} \theta^{(\ell)}$. Before proceeding further, Eq. (35) requires some explanation. In Sec. 2.1.3, we have introduced $\delta_{v}$ in terms of the Lie derivative on a tensorial object. However, the expansion $\theta^{(\ell)}$ is not a scalar quantity in the sense of a point-like (tensorial) field defined on the manifold $\mathcal{M}$. The expansion is a quasi-local object whose very definition at a point $p \in \mathcal{M}$ requires the choice of a (portion of a) surface $\mathcal{S}$ passing through $p$. In this sense, $\delta_{\gamma v}$ (with $\gamma$ a function on $\mathcal{S}$ ) cannot be in general evaluated as 
a Lie derivative. Consider a displacement of the surface $\mathcal{S}_{t}$ by a vector $\gamma v^{a}$. The surface $\mathcal{S}_{t+\delta t}$ and therefore $\left.\theta^{(\ell)}\right|_{t+\delta t}$ depend on the angular dependence of $\gamma$, so that $\delta_{\gamma v} \theta^{(\ell)} \neq \gamma \delta_{v} \theta^{(\ell)}$. The operator $\delta_{v}$ still satisfies a linear property for constant linear combinations, $\delta_{a v+b w} \theta^{(\ell)}=a \delta_{v} \theta^{(\ell)}+b \delta_{w} \theta^{(\ell)}(a, b \in \mathbb{R})$, and the Leibnitz rule, $\delta_{v}\left(\gamma \theta^{(\ell)}\right)=\left(\delta_{v} \gamma\right) \theta^{(\ell)}+\gamma \delta_{v} \theta^{(\ell)}$. Details about this operator can be found in Refs. $30,24,43 .^{\mathrm{g}}$ Here we rather exploit a practical trick for the evaluation of $\delta_{\gamma v} \theta^{(\ell)}$, based on the remark that given the vector $v^{a}$ normal to $\mathcal{S}$, and not multiplied by a function on $\mathcal{S}$, it still holds formally $\delta_{v} \theta^{(\ell)}=\mathcal{L}_{v} \theta^{(\ell)}$. Then, we can evaluate $\delta_{\gamma v} \theta^{(\ell)}$ as $\delta_{\gamma v} \theta^{(\ell)}=\delta_{\tilde{v}} \theta^{(\ell)}=\mathcal{L}_{\tilde{v}} \theta^{(\ell)}$, with $\tilde{v}^{a}=\gamma v^{a}$. In particular, the application of this strategy to the second line of (35) goes as follows. We write $\tilde{k}^{a}=\beta k^{a}$ and calculate $\delta_{\tilde{k}} \theta^{(\ell)}$ through a Lie derivative evaluation. This results in

$$
\delta_{\tilde{k}} \theta^{(\ell)}=\left(-\tilde{k}^{c} \ell_{c}\right)\left[{ }^{2} D^{c} \Omega_{c}^{(\tilde{k})}+\Omega_{c}^{(\tilde{k})} \Omega^{(\tilde{k})^{c}}-\frac{1}{2}{ }^{2} R\right]+8 \pi T_{a b} \tilde{k}^{a} \ell^{b}
$$

Using $\left(-\tilde{k}^{c} \ell_{c}\right)=\beta, \Omega_{a}^{(\tilde{k})}=\Omega_{a}^{(k)}+{ }^{2} D_{a} \ln \beta$ and $\Omega_{a}^{(k)}=-\Omega_{a}^{(\ell)}$ the expression for $\delta_{\tilde{k}} \theta^{(\ell)}$ in (35) follows (cf. footnote b).

\subsubsection{Generic properties of dynamical FOTHs}

We review some generic properties of dynamical trapping horizons. ${ }^{14,24,26,32,44}$

(i) Topology law: under the dominant energy condition, sections $\mathcal{S}_{t}$ are topological spheres. This can be shown by integrating $\delta_{k} \theta^{(\ell)}<0$ on $\mathcal{S}_{t}$. Under the assumed energy condition, the Euler characteristic $\chi$

$$
\chi=\frac{1}{4 \pi} \int_{\mathcal{S}}^{2} R^{2} \epsilon=\frac{1}{2 \pi} \int_{\mathcal{S}}\left(-\delta_{k} \theta^{(\ell)}+\Omega_{c}^{(\ell)} \Omega^{(\ell)^{c}}+8 \pi T_{a b} k^{a} \ell^{b}\right)^{2} \epsilon,
$$

is positive and, being $\mathcal{S}_{t}$ a closed 2-surface, its spherical topology follows.

(ii) Signature law: under the null energy condition, $\mathcal{H}$ is completely partitioned into null worldtube sections (where $\delta_{\ell} \theta^{(\ell)}=0$ ) and space-like worldtube sections (where $\delta_{\ell} \theta^{(\ell)} \neq 0$ at least on a point). Applying a maximum principle to the trapping horizon constraint condition, Eq. (37), it follows that either $C=$ const $\geq 0$, or $C$ is a function $C>0$ everywhere on $\mathcal{S}$ (cf. discussion in Sec. 2.4).

(iii) Area law: under the null energy condition, if $\delta_{\ell} \theta^{(\ell)} \neq 0$ somewhere on $\mathcal{S}_{t}$, the area grows locally everywhere on $\mathcal{S}_{t}$. Otherwise the area in constant along the evolution. This follows from applying the future condition, $\theta^{(k)}<0$, and the signature law to $\delta_{h}^{2} \sqrt{q}=-C \theta^{(k)} \sqrt{q}$ [cf. Eq. (17)].

(iv) Preferred choice of null tetrad on a DH. According to the foliation uniqueness and existence results discussed in Sec. 3.2.1, there is a unique evolution vector

gee also the treatment in terms of Lie derivatives in the double null foliations treatment in Refs. 14 and 23. 
$h^{a}$ tangent to $\mathcal{H}$ and orthogonal to $\mathcal{S}_{t}$, such that $h^{a}$ transports $\mathcal{S}_{t} \in \Sigma_{t}$ onto $\mathcal{S}_{t+\delta t} \in \Sigma_{t+\delta t}$ : that is, $\delta_{h} t=1$, for a given function $t$ defining a $3+1$ spacetime foliation $\left\{\Sigma_{t}\right\}$. Denoting the unit timelike normal to $\Sigma_{t}$ by $n^{a}$, the lapse function by $N$, i.e. $n_{a}=-N \nabla_{a} t$, and the normal to $\mathcal{S}_{t}$ tangent to $\Sigma_{t}$ by $s^{a}$, we can write on the horizon $\mathcal{H}_{N}$

$$
h^{a}=N n^{a}+b s^{a},
$$

for some $b$ fixed from $N$ and $C$ in $(16)$, as $2 C=(b+N)(b-N)$. The expression of the evolution vector as $h^{a}=\ell^{a}-C k^{a}$ [cf. Eq. (16)] links the scaling of $\ell^{a}$ and $k^{a}$ to that of $h^{a}$. In particular, $\ell^{a}$ is singled out as the only null normal to $\mathcal{S}_{t}$ such that $h^{a} \rightarrow \ell^{a}$ as the trapping horizon is driven to stationarity $(C \rightarrow 0 \Leftrightarrow$ $\left.\delta_{\ell} \theta^{(\ell)} \rightarrow 0\right)$. Writing generically the null normals at $\mathcal{H}_{N}$ as $\ell^{a}=f \cdot\left(n^{a}+s^{a}\right)$ and $k^{a}=\left(n^{a}-s^{a}\right) /(2 f)$, Eqs. (40) and (16) lead to a preferred scaling of null normals on the $\mathrm{DH} \mathcal{H}_{N}$

$$
\ell_{N}^{a}=\frac{N+b}{2}\left(n^{a}+s^{a}\right), \quad k_{N}^{a}=\frac{1}{N+b}\left(n^{a}-s^{a}\right) .
$$

\subsubsection{Geometric balance equations}

One of the main motivations for the development of quasi-local horizon formalisms is the extension of the laws of black hole thermodynamics to dynamical regimes. This involves in particular finding balance equations to control the rate of change of physical quantities on the horizon, in terms of appropriate fluxes through the hypersurface. This is an extensive subject whose review is beyond our scope. In the spirit of the present discussion, we restrain ourselves to comment on the balance equations for two geometric quantities on $\mathcal{S}_{t}$ : the area $A=\int_{\mathcal{S}} d A=\int_{\mathcal{S}}{ }^{2} \epsilon$ and the angular momentum $J[\phi]$ in Eq. (15), for an axial Killing (or, more generally, divergence-free) vector $\phi^{a}$. That is, we aim at writing

$$
\frac{d A}{d t}=\int_{\mathcal{S}_{t}} F^{A} d A, \quad \frac{d J[\phi]}{d t}=\int_{\mathcal{S}_{t}} F^{J} d A,
$$

for appropriate area $F^{A}$ and angular momentum $F^{J}$ fluxes, with $d / d t$ associated to the foliation Lie-transported by $h^{a}$. Eventually, one would aim at writing a first law of thermodynamics by appropriately combining the previous balance equations

$$
\kappa_{t} \frac{d A}{d t}+\Omega_{t} \frac{d J[\phi]}{d t}=\int_{\mathcal{S}_{t}} F^{E} d A
$$

for some functions $\kappa_{t}$ and $\Omega_{t}$ on $\mathcal{S}_{t}$, so that $F^{E}$ is interpreted as an energy flux. ${ }^{24,26,44-50}$ As a first step towards (42) we write evolution equations for the expansion $\theta^{(h)}$ and the 1-form $\Omega_{a}^{(\ell)}$ along the evolution vector $h^{a}$. These equations are given by the projection of some of the components of the Einstein equations onto $\mathcal{H}$. Introducing a 4-momentum current density $p_{a}=-T_{a b} \tau^{b}$, with $\tau^{a}$ the vector 
orthogonal to $\mathcal{H}$ defined in (16), such equations provide three of the components of $p_{a}$. The fourth is given by the trapping horizon condition (38). In brief:

(i) Evolution element of area ${ }^{51,52}\left(p_{a} h^{a}=-T_{a b} \tau^{b} h^{a}\right)$ :

$$
\begin{aligned}
\left(\delta_{h}+\theta^{(h)}\right) \theta^{(h)}= & -\kappa^{(h)} \theta^{(h)}+\sigma_{a b}^{(h)} \sigma^{(\tau)^{a b}} \\
& +\frac{\left(\theta^{(h)}\right)^{2}}{2}-2^{2} D^{a} Q_{a}+8 \pi T_{a b} \tau^{a} h^{b}-\frac{\theta^{(k)}}{8 \pi} \delta_{h} C,
\end{aligned}
$$

with $Q_{a}=\frac{1}{4 \pi}\left[C \Omega_{a}^{(\ell)}-1 / 2^{2} D_{a} C\right]$ and $\kappa^{(h)}=-h^{b} k^{c} \nabla_{b} \ell_{c}$.

(ii) Evolution normal (rotation) form ${ }^{23,52} \Omega_{a}^{(\ell)}\left(p_{b} q_{a}^{b}=-T_{b c} \tau^{c} q_{a}^{b}\right)$ :

$$
\left(\delta_{h}+\theta^{(h)}\right) \Omega_{a}^{(\ell)}={ }^{2} D_{a} \kappa^{(h)}-{ }^{2} D^{c} \sigma_{a c}^{(\tau)}-{ }^{2} D_{a} \theta^{(h)}+8 \pi q^{b}{ }_{a} T_{b c} \tau^{c}-\theta^{(k) 2} D_{a} C .
$$

(iii) Normal component $\left(p_{a} \tau^{a}=-T_{a b} \tau^{b} \tau^{a}\right)$ : linear combination, using $\tau^{a}=$ $2 \ell^{a}-h^{a}$, of $T_{a b} \tau^{a} h^{b}$ (area element evolution) and $T_{a b} \tau^{a} \ell^{b}$ [trapping horizon constraint (38)].

In order to derive the evolution equation for $A$, we write $A=\int_{\mathcal{S}} d A=\int_{\mathcal{S}}{ }^{2} \epsilon$ so that, using the transport of $\mathcal{S}_{t}$ into $\mathcal{S}_{t+\delta t}$ by $h^{a}$, we have $\frac{d A}{d t}=\int_{\mathcal{S}} \delta_{h}(d A)=\int_{\mathcal{S}} \theta^{(h)} d A$ and $\frac{d^{2} A}{d t^{2}}=\int_{\mathcal{S}}\left(\delta_{h} \theta^{(h)}+\left(\theta^{(h)}\right)^{2}\right) d A$. From Eq. (44) it then follows

$$
\frac{d^{2} A}{d t^{2}}+\bar{\kappa}^{\prime} \frac{d A}{d t}=\int_{\mathcal{S}_{t}}\left[8 \pi T_{a b} \tau^{a} h^{b}+\sigma_{a b}^{(h)} \sigma^{(\tau)^{a b}}+\frac{\left(\theta^{(h)}\right)^{2}}{2}+\left(\bar{\kappa}^{\prime}-\kappa^{\prime}\right) \theta^{(h)}\right]{ }^{2} \epsilon,
$$

where $\kappa^{\prime} \equiv \kappa-\delta_{h} \ln C$ and $\bar{\kappa}^{\prime}=\bar{\kappa}(t) \equiv A^{-1} \int_{\mathcal{S}_{t}} \kappa^{\prime 2} \epsilon$. Note that this is a secondorder equation for the area. ${ }^{51}$ Near equilibrium, the second time derivative as well as higher-order terms can be neglected leading to the Hawking and Hartle expression ${ }^{53}$

$$
\bar{\kappa}^{\prime} \frac{d A}{d t}=\int_{\mathcal{S}_{t}}\left[8 \pi T_{a b} \ell^{a} \ell^{b}+\sigma_{a b}^{(\ell)} \sigma^{(\ell)}{ }^{a b}\right] d A .
$$

Regarding the evolution equation for $J[\phi]$, we make use of Eq. (45) together with a divergence-free condition on $\phi^{a}$ (that relaxes the Killing condition) and the condition that $\phi^{a}$ is Lie-dragged by the evolution vector $h^{a}$. Then ${ }^{23,54,55}$

$$
\frac{d}{d t} J(\phi)=-\int_{\mathcal{S}_{t}} T_{a b} \tau^{a} \phi^{b 2} \epsilon-\frac{1}{16 \pi} \int_{\mathcal{S}_{t}} \sigma_{a b}^{(\tau)} \delta_{\phi} q^{a b 2} \epsilon
$$

with the second term on the right-hand side accounting for a non-Killing $\phi^{a}$. Interestingly in dynamical (spacelike) horizons $\mathcal{H}$, the conditions ${ }^{2} D_{a} \phi^{a}=0$ and $\delta_{h} \phi^{a}$ completely fix ${ }^{54}$ the form of the vector $\phi^{a}: \phi^{a}={ }^{2} \epsilon^{a b 2} D_{b} \theta^{(h)}$. 


\subsubsection{Open geometric issues and physical remarks}

To close this generic section on geometric aspects of dynamical horizons, we list some relevant open geometric problems:

(i) Canonical choice of dynamical trapping horizon. DHs are highly non-unique in a given black hole spacetime. A natural question concerns the possibility of making a canonical choice. There has been some attempts in this direction based on entropic arguments. ${ }^{51,56-58}$ A very interesting avenue lies on the recently introduced notion of the core of the trapped region ${ }^{21}$ (see also J. M. M. Senovilla's contribution ${ }^{15}$ ).

(ii) Asymptotics of dynamical horizons to the event horizon. One would expect DHs to asymptote generically to the event horizon at late times. This is indeed a topic of active research. ${ }^{16,59-61}$

(iii) Black hole singularity covering by dynamical horizons. In addition to the asymptotics of DHs to the event horizon, it is also of interest to assess their behaviour at the birth of the black hole singularity, in particular their capability to separate (dress) singularities from the rest of the spacetime (see Sec. 5.4.4).

DHs as physical surfaces. Dynamical horizons are objects with very interesting geometric properties for the study of black hole spacetimes. In addition, from a physical perspective it is remarkable that they admit a nontrivial thermodynamical description (cf. A. Nielsen's contribution ${ }^{22}$ ). However, it is also important to underline that, if thought as boundaries of compact physical objects (in the sense we think, say, of the surface of a neutron star), then they have nonstandard physical properties:

(a) They are non-unique. From an Initial Value Problem perspective, the question about the evolution of a given $\mathrm{AH}$ is not well-posed, since it depends on the $3+1$ slicing choice (such non-uniqueness in evolution is typical in gauge dynamics).

(b) Dynamical trapping horizons are superluminal, something difficult to reconciliate with the physical surface of an object.

(c) DHs show a nonlocal behavior. For instance, they grow globally (reacting as a whole) when energy crosses them at a given local region (even a point). This is a consequence of their intrinsic elliptic, rather than hyperbolic, behavior.

\section{Black Hole Spacetimes in an Initial-Boundary Value Problem Approach}

In the context of an Initial-Boundary Value Problem approach to the construction of spacetimes, dynamical trapping horizons play a role at two levels: (i) first, as an a priori ingredient to be incorporated into a given PDE formulation of Einstein equations, and (ii) as an a posteriori tool to extract information of the constructed spacetimes. In this section we address their application as an a priori ingredient. 


\subsection{The initial value problem in general relativity: $3+1$ formalism}

Our general basic problem is the control ${ }^{62}$ of the qualitative and quantitative aspects of generic solutions to Einstein equations in dynamical scenarios involving a black hole spacetime. The Initial-Boundary Value Problem approach provides a powerful avenue to it. Such a strategy is well suited, on the one hand, to the use of global analysis and Partial Differential Equations (PDE) tools for controlling the qualitative aspects of the problem and, on the other hand, to the employment of numerical techniques to assess the quantitative ones. In particular, we focus here on the Cauchy (and hyperboloidal) Initial Value Problem.

\subsubsection{Einstein equations: Constraint and evolution system}

General Relativity is a geometric theory in which not all the fields constitute physical degrees of freedom (gauge theory), so that constraints among the fields are present. In the passage from the geometric formulation of the theory to an analytic problem in the form of a specific PDE system, several PDE subsystems enter into scene. ${ }^{63}$ First, the constraint system is determined by the (Gauss-Codazzi) conditions that data on a three-dimensional Riemannian manifold must satisfy to be considered as initial data on a spacetime slice. The Hamiltonian and momentum constraints are determined by the $G_{a b} n^{b}$ components of the Einstein equation, where $n^{a}$ is a unit timelike vector normal to the initial slice. Second, the evolution system is built from the rest of Einstein equation, including possible auxiliary fields. The gauge system determines the dynamical choice of coordinates in the spacetime. Finally, a subsidiary system controls the internal consistency of the previous systems.

\subsection{2. $3+1$ formalism}

We introduce some notation regarding the $3+1$ formalism. ${ }^{64}$ As in Sec. 3.2.4, given a $3+1$ slicing of spacetime by spacelike hypersurfaces $\left\{\Sigma_{t}\right\}$, the unit timelike normal to $\Sigma_{t}$ is denoted by $n^{a}$ and the lapse function as $N, n_{a}=-N \nabla_{a} t$, with $t$ the scalar function defining the $3+1$ slicing. The $3+1$ evolution vector is denoted by $t^{a}=N n^{a}+\beta^{a}$, where $\beta^{a}$ is the shift vector. The induced metric on $\Sigma_{t}$ is denoted by $\gamma_{a b}$, i.e. $\gamma_{a b}=g_{a b}+n_{a} n_{b}$. We choose the following sign convention for the extrinsic curvature of $\Sigma_{t}$ in $\mathcal{M}: K_{a b}=-\gamma^{c}{ }_{a} \nabla_{c} n_{b}=-\frac{1}{2} \mathcal{L}_{n} \gamma_{a b}$. In particular, we can write $K_{i j}=\frac{1}{2 N}\left(\gamma_{i k} D_{j} \beta^{k}+\gamma_{j k} D_{i} \beta^{k}-\dot{\gamma}_{i j}\right)$, where the dot denotes the derivative $\mathcal{L}_{t}$. Indices $i, j, k, \ldots$ are used for objects leaving on $\Sigma_{t}$. For concreteness, we focus on a particular $3+1$ decomposition of Einstein equations, namely involving the following conformal decomposition (conformal Ansatz ${ }^{65}$ ) for data $\left(\gamma_{i j}, K^{i j}\right)$ on $\Sigma_{t}$ :

$$
\gamma_{i j}=\Psi^{4} \tilde{\gamma}_{i j}, \quad K_{i j}=\Psi^{\zeta} \tilde{A}_{i j}+\frac{1}{3} K \gamma_{i j}
$$


for several $\zeta$ choices. Denoting by $\tilde{D}_{i}$ the Levi-Civita connection associated with $\tilde{\gamma}_{i j}$ and inserting (48) into Einstein equations leads to a coupled elliptic-hyperbolic PDE system on the variables $\Psi, \beta^{i}, N$ and $\tilde{\gamma}_{a b}$. The elliptic part has the form

$$
\begin{aligned}
\tilde{D}_{k} \tilde{D}^{k} \Psi-\frac{{ }^{3} \tilde{R}}{8} \Psi & =S_{\Psi}\left[\Psi, N, \beta^{i}, K, \tilde{\gamma}, \ldots\right], \\
\tilde{D}_{k} \tilde{D}^{k} \beta^{i}+\frac{1}{3} \tilde{D}^{i} \tilde{D}_{k} \beta^{k}+{ }^{3} \tilde{R}_{k}^{i} \beta^{k} & =S_{\beta}\left[\Psi, N, \beta^{i}, K, \tilde{\gamma}, \ldots\right], \\
\tilde{D}_{k} \tilde{D}^{k} N+2 \tilde{D}_{k} \ln \Psi \tilde{D}^{k} N & =S_{N}\left[N, \Psi, \beta^{i}, K, \tilde{\gamma}, \dot{K}, \ldots\right],
\end{aligned}
$$

where the equation on $\Psi$ follows from the Hamiltonian constraint, the equation on $\beta^{i}$ follows from the momentum constraint and the third equation on $N$ follows from a (gauge) condition imposed on $\dot{K}$. If only solved on an initial slice with $\tilde{\gamma}_{i j}, \dot{\tilde{\gamma}}^{i j}, K$ and $\dot{K}$ as free data, this system constitutes the Extended Conformal Thin Sandwich approach to initial data. ${ }^{66,67}$ If we solve it during the whole evolution, together with

$$
\frac{\partial^{2} \tilde{\gamma}^{i j}}{\partial t^{2}}-\frac{N^{2}}{\Psi^{4}} \Delta \tilde{\gamma}^{i j}-2 \mathcal{L}_{\beta} \frac{\tilde{\gamma}^{i j}}{\partial t}+\mathcal{L}_{\beta} \mathcal{L}_{\beta} \tilde{\gamma}^{i j}=S_{\tilde{\gamma}}^{i j}\left[N, \Psi, \beta^{i}, K, \tilde{\gamma}, \ldots\right]
$$

for $\tilde{\gamma}_{i j}$, it defines a particular constrained evolution formalism. ${ }^{68-70}$

\subsection{Initial data: Isolated horizon inner boundary conditions}

There are two standard approaches to ensure that initial data on a slice $\Sigma_{0}$ correspond to a black hole spacetime. The punctures approach exploits the nontrivial topology ${ }^{71,72}$ of $\Sigma_{0}$, whereas the excision approach removes a sphere from the initial slice and enforces it to be inside the black hole region. In a sense, they both reflect the global versus quasi-local discussion in Sec. 1. Here we discuss the use of inner boundary conditions derived from the IH formalism, when constructing initial data of black holes instantaneously in equilibrium in an excision approach.

\subsubsection{Non-expanding horizon conditions}

The NEH condition $\Theta_{a b}^{(\ell)}=0$ in Eq. (26) [or (25)] provides three inner boundary conditions for the elliptic system (49). In particular, they enforce the excised surface $\mathcal{S}_{0}$ to be a section of a quasi-local horizon instantaneously in equilibrium.

For a given choice of free initial data in system (49), the geometric NEH inner boundary conditions, $\Theta_{a b}^{(\ell)}=0$, must be complemented with two additional inner boundary (gauge) conditions. Denoting by $s^{i}$ the normal vector to $\mathcal{S}_{t}$ tangent to $\Sigma_{t}$, we write $\beta^{i}=\beta^{\perp} s^{i}+\beta_{\|}^{i}$, with $\beta^{\perp}=\beta^{i} s_{i}$ and $\beta_{\|}^{i} s_{i}=0$. Adapting the coordinate system to the horizon (i.e. $t^{a}=\ell^{a}+\beta_{\|}^{a} \Leftrightarrow \beta^{\perp}=N$ ) supplies a fourth gauge condition 
that, together with the $\theta^{(\ell)}=0$ and $\sigma_{a b}^{(\ell)}=0 \mathrm{NEH}$ conditions, reads ${ }^{28,73-75}$

$$
\begin{aligned}
& \tilde{s}^{i} \tilde{D}_{i} \Psi+\tilde{D}_{i} \tilde{s}^{i} \Psi+\Psi^{-1} K_{i j} \tilde{s}^{i} \tilde{s}^{j}-\Psi^{3} K=0, \\
& { }^{2} \tilde{D}_{a} \tilde{\beta}_{b}^{\|}+{ }^{2} \tilde{D}_{b} \tilde{\beta}_{a}^{\|}-\left({ }^{2} \tilde{D}_{c} \beta_{\|}^{c}\right) \tilde{q}_{a b}=0, \quad \beta^{\perp}=N,
\end{aligned}
$$

where $\tilde{q}_{a b}=\Psi^{4} q_{a b}$ and $\tilde{\beta}_{a}^{\|}=\tilde{q}_{a b} \beta^{\|^{b}}$. A fifth boundary condition, namely for $N$, can be obtained by choosing a slicing inner boundary condition. The (gauge) weakly isolated horizon structure can be used in this sense. ${ }^{28,76}$

\subsection{2. (Full) isolated horizon conditions}

The next geometric quasi-equilibrium horizon structure is a (full) IH (cf. Secs. 3.1.4 and 3.1.5). This involves three additional conditions that cannot be accommodated in system (49) for fixed free initial data. However, we can revert the argument and employ IH conditions to determine improved quasi-equilibrium free initial data $\tilde{\gamma}_{a b}$ and $\dot{\tilde{\gamma}}_{a b}$ by solving the full set of Einstein equations (49) and (50) under a quasi-equilibrium Ansatz. Namely, we can set $\partial_{t} \tilde{\gamma}^{a b}$ and $\frac{\partial^{2} \tilde{\gamma}^{a b}}{\partial t^{2}}$ in (50) to prescribed functions $f_{1}^{a b}$ and $f_{2}^{a b}$ and consider the elliptic system formed by (49) together with

$$
-\frac{N^{2}}{\Psi^{4}} \tilde{\Delta} \tilde{\gamma}^{a b}+\mathcal{L}_{\beta} \mathcal{L}_{\beta} \tilde{\gamma}^{a b}=S_{\tilde{\gamma}}^{a b}-f_{2}^{a b}+2 \mathcal{L}_{\beta} f_{1}^{a b} .
$$

This extended elliptic system is solved for ten fields: $\left(\Psi, \beta^{a}, N\right)$ and the five $\tilde{\gamma}^{a b}$. Geometrically, we need to impose four gauge inner conditions, leaving exactly six inner conditions to be fixed. Remarkably, this fits exactly the six IH conditions ${ }^{77}$

$$
\Theta_{a b}^{(\ell)}=0, \quad \Theta_{a b}^{(k)}=\Theta_{a b}^{(k)}\left(\kappa_{o}, \tilde{q}_{a b}, \Omega_{a}^{(\ell)}\right) \Leftrightarrow F_{a b}^{\Theta^{(k)}}\left(\kappa_{o}, \Psi, \beta^{a}, N, \tilde{\gamma}_{a b}\right)=0,
$$

where $F_{a b}^{\Theta^{(k)}}$ is determined by the expression for $\Theta_{a b}^{(k)}$ in Eq. (31), fixed up to the value of the constant $\kappa_{O}$. It is interesting to remark that this $\mathrm{IH}$ prescription ${ }^{77}$ completely fixes (up a $\kappa_{o}$ one-parameter family) the extrinsic curvature tensor $\mathcal{K}_{a b}^{c}=$ $k^{c} \Theta_{a b}^{(\ell)}+\ell^{c} \Theta_{a b}^{(k)}\left[\right.$ cf. Eq. (8)] of $\mathcal{S}_{0}$ as embedded in the spacetime $\mathcal{M}$.

\subsection{Constrained evolutions: Trapping horizon inner boundary conditions}

The elliptic-hyperbolic system (49)-(50) provides a constrained evolution scheme for the dynamical construction of the spacetime. Adopting an excision approach to black holes, we need five inner boundary conditions for the elliptic part of the system. In principle, dynamical trapping horizon conditions on the inner boundary worldtube $\mathcal{H}=\cup_{t} \mathcal{S}_{t}$ provide a geometric prescription guaranteeing that $\mathcal{H}$ remains in the black hole region. However, imposing FOTH conditions on $\mathcal{H}$ can be too stringent in generic evolutions. The reason is that the constructed worldtube of MOTS $\mathcal{H}$, regarded as a hypersurface in spacetime, can change signature. This is in 
conflict with the outer condition in Sec. 2.2 (something related to jumps occurring generically $^{78-80}$ in $\mathrm{AH}$ evolutions; see Sec. 5.1.1) so that the resulting PDE system can become ill-posed. In this context, trapping horizon conditions together with the requirement of recovering $\mathrm{NEH}$ inner conditions at the equilibrium limit, provide an appropriate relaxed set of inner boundary conditions. ${ }^{81}$ More specifically, trapping horizon conditions provides two geometric conditions $\theta^{(\ell)}=0$ and $\delta_{h} \theta^{(\ell)}=0$, whereas three additional gauge conditions guarantee the recovery of $\mathrm{NEH}$ at equilibrium.

As a first step, as in Sec. 4.2.1, we choose a coordinate system adapted to the horizon. This means that spacetime evolution $t^{a}$ is tangent to $\mathcal{H}$. Decomposing the shift as $\beta^{a}=\beta^{\perp} s^{a}+\beta_{\|}^{a}$, then $t^{a}$ is written as $t^{a}=N n^{a}+\beta^{a}=\left(N n^{a}+b s^{a}\right)+$ $\beta_{\|}^{a}+\left(\beta^{\perp}-b\right) s^{a}=h^{a}+\beta_{\|}^{a}+\left(\beta^{\perp}-b\right) s^{a}$. Therefore $t^{a}$ is tangent to $\mathcal{H}$ if and only if $\beta^{\perp}=b$.

(i) Geometric trapping horizon conditions. Condition $\theta^{(\ell)}=0$ leads, in terms of the $3+1$ quantities in Sec. 4.1.2, to the expression in the first line of Eq. (51). Condition $\delta_{h} \theta^{(\ell)}=0$ in Eq. (38), using the adapted coordinate system $\beta^{\perp}=b$, leads to

$$
\left[-{ }^{2} D_{a}^{2} D^{a}-2 L^{a 2} D_{a}+A\right]\left(\beta^{\perp}-N\right)=B\left(\beta^{\perp}+N\right),
$$

where $L_{a}=K_{i j} s^{i} q_{a}^{j}, A=\frac{1}{2}{ }^{2} R-{ }^{2} D_{a} L^{a}-L_{a} L^{a}-4 \pi T_{a b}\left(n^{a}+s^{a}\right)\left(n^{b}-s^{b}\right)$, and $B=\frac{1}{2} \sigma_{a b}^{(\hat{\ell})} \sigma^{(\hat{\ell}) a b}+4 \pi T_{a b}\left(n^{a}+s^{b}\right)\left(n^{b}+s^{b}\right)$, with $\hat{\ell}^{a}=n^{a}+s^{a}$.

(ii) Gauge boundary conditions I. Aiming at recovering NEH boundary conditions for $\beta_{\|}^{a}$, we first express $\delta_{h} q_{a b}=\theta^{(h)} q_{a b}+2 \sigma_{a b}^{(h)}$ in adapted coordinates $\left(h^{a}=\right.$ $\left.t^{a}-\beta_{\|}^{a}\right)$

$$
2 \sigma_{a b}^{(h)}=\left(\frac{\partial q_{a b}}{\partial t}-\frac{\partial}{\partial t} \ln \sqrt{q} q_{a b}\right)-\left({ }^{2} D_{a} \beta_{b}^{\|}+{ }^{2} D_{b} \beta_{a}^{\|}-{ }^{2} D_{c} \beta_{\|}^{c} q_{a b}\right) .
$$

Then, the coordinate choice $\partial_{t} q_{a b}-\partial_{t} \ln \sqrt{q} q_{a b}=0$ leads to the condition on $\beta_{a}^{\|}$

$$
{ }^{2} D_{a} \beta_{b}^{\|}+{ }^{2} D_{b} \beta_{a}^{\|}-{ }^{2} D_{c} \beta_{\|}^{c} q_{a b}=-2 \sigma_{a b}^{(h)},
$$

that is completed by using the evolution equation for $\sigma_{a b}^{(h)}$ on $\mathcal{H}$

$$
\begin{aligned}
\delta_{h} \sigma_{a b}^{(h)}= & -q_{a}^{d} q_{b}^{f} C^{c}{ }_{d e f} \ell_{c} \ell^{e}-C^{2} q_{a}^{d} q_{b}^{f} C^{c}{ }_{d e f} k_{c} k^{e} \\
& -8 \pi C\left[q_{a}^{c} q_{b}^{d} T_{c d}-\frac{1}{2}\left(q^{c d} T_{c d}\right) q_{a b}\right]+\cdots .
\end{aligned}
$$

(iii) Gauge boundary conditions II. The slicing condition for $N$ is essentially free. However, from Properties 1 and 2 in Sec. 3.2.1, such a choice is equivalent to choosing a dynamical horizon $\mathcal{H}$. Since each $\mathcal{H}$ is a genuine geometric object, this suggests the possibility of recasting into geometric terms the gauge choice of inner boundary condition for $N$, by selecting a trapping horizon $\mathcal{H}$ satisfying 
some specific geometric criterion for $\mathcal{H}$. As an example of this, maximizing the area growth rate $\dot{A}$ of $\mathcal{H}$ leads ${ }^{51,81}$ to the condition $\beta^{\perp}-N=-$ const. $\cdot \theta^{(\hat{k})}$, with $\hat{k}^{a}=n^{a}-s^{a}$.

\section{A posteriori Analysis of Black Hole Spacetimes}

We address here the application of dynamical trapping horizons to the a posteriori analysis of spacetimes, their main application in the Initial Value Problem approach.

\section{1. "Tracking" the black hole region: $A H$ finders}

As discussed in Sec. 1.2, event horizons cannot be located during the spacetime evolution. However, in applications such as numerical relativity, assessing if a region of spacetime lays inside the black hole region can be crucial during the evolution. Under the assumption of cosmic censorship, the location of AHs in spatial sections $\Sigma_{t}$ and the worldtubes constructed by piling them up (see Sec. 1.3.1) are extremely useful to determine the evolutive properties of the black hole. In this sense, $A H$ finders prove to be extraordinary practical tools. These are algorithms for searching surfaces $\mathcal{S}_{t} \subset \Sigma_{t}$ that satisfy the MOTS condition $\theta^{(\ell)}=0$. There are many approaches to this problem, ${ }^{82}$ but all of them aim at solving the condition $D_{i} s^{i}-K+K_{i j} s^{i} s^{j}=0$. For instance, assuming spherical topology, we can characterize the surface in an adapted (spherical) coordinate system as $F(r, \theta, \varphi)=r-h(\theta, \varphi)$ with $F=$ const, so that the normal vector to $\mathcal{S}_{t}$ is given by $s_{i}=\frac{1}{\sqrt{D^{i} F \cdot D_{i} F}} D_{i} F$ with $D_{i} F=\left(1,-\partial_{\theta} h,-\partial_{\varphi} h\right)$ in the spherical coordinate system. The MOTS condition becomes then a nonlinear elliptic equation on $h$ that can be solved very efficiently.

\subsubsection{Understanding AH jumps}

Noncontinuous jumps of AHs occur generically in $3+1$ black hole evolutions. The dynamical trapping horizon framework sheds light ${ }^{79,80,83}$ on these $A H$ jumps, suggesting a spacetime picture where the jumps are understood as multiple spatial cuts of a single underlying spacetime MOTS worldtube. Jumps are associated with the change of metric type of the horizon hypersurface (see Fig. 2). This is particularly dramatic in binary black hole simulations, where at a given time $t$ the two individual nonconnected horizons jump to a common one. A specific prediction of the dynamical horizon picture is that new (common) horizons form in pairs ${ }^{37,83}$ : the outermost (apparent) horizon growing in area and a dual inner one whose area decrease in the time $t$. Apart from providing a better understanding of the underlying geometry of the trapped region, this spacetime picture can be of use in the study of flows interpolating between a given MOTS and the eventual event horizon, something of potential interest for studies of the Penrose inequality (see Sec. 5.2.2). 


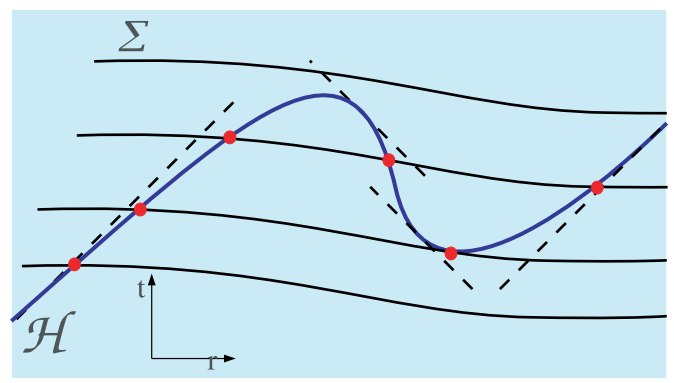

Fig. 2. (Color online) Illustration of AH jumps as multiple cuts of a single spacetime MOTSworltube $\mathcal{H}$. In particular, timelike sections of $\mathcal{H}$ produce jumps (null hypersurfaces are represented with $\left.45^{\circ}\right)$.

\subsection{Horizon analysis parameters}

Assigning parameters to (individual) black holes can offer crucial insight into the dynamical evolution. These can be physical parameters like the mass or the angular momentum, or diagnosis parameters informing of relevant dynamical properties. Given the generic absence of background rigid structures, first-principles parameters are often out of reach and one must follow nonrigorous or pragmatic approaches.

\subsubsection{Mass and angular momentum. IH and DH multipoles}

In our discussion we have avoided entering into first-principles physical issues, stressing rather the geometric properties of dynamical trapping horizons and their applications. However, mass and angular momentum estimates for individual black holes, either fundamental or effective, are extremely important in the modeling of astrophysical systems involving matter or binary systems. The problem has two aspects. First, one must identify a surface to be associated with the black hole boundary. Discussion in Sec. 1 shows that this is a delicate question. In any case, AHs provide surfaces $\mathcal{S}_{t} \in \Sigma_{t}$ tracking the black hole region, that can be employed as preferred choices for pragmatic estimations. The second problem refers to the ambiguities in the quasi-local characterization of the gravitational field mass and angular momentum in General Relativity. ${ }^{84,85}$ Regarding the angular momentum, the Komar expression (15) characterizes appropriately the axisymmetric case. Effective prescriptions $^{86-88}$ exist for generic horizons. Regarding the mass, the irreducible mass $M_{\text {irred }} A=16 \pi M_{\text {irred }}^{2}$ provides a purely geometric estimation in terms of the area. Its physical interpretation as the portion of the black hole mass that cannot be extracted by a Penrose process, together with its equivalence with the Hawking energy, $M_{\text {Hawking }}=\sqrt{A /(16 \pi)}\left(1+1 /(8 \pi) \oint \theta^{(\ell)} \theta^{(k)} d A\right)$ for MOTSs, makes it useful in numerical applications and in thermodynamical treatments. ${ }^{45,46}$ Given $A$ and $J$ one can also consider ${ }^{32}$ the Christodoulou expression for the Kerr mass

$$
M_{\mathrm{Chris}}=\left(\frac{A}{16 \pi}+\frac{4 \pi J^{2}}{A}\right)^{\frac{1}{2}}
$$


There are many prescriptions for the quasi-local mass. ${ }^{84,85}$ It is therefore crucial to choose and keep consistently a prescription when comparing different solutions. In this latter sense, the mass and angular momentum horizon geometric multipoles $I_{n}$ and $L_{n}$ in (34) offer a useful and refined diagnosis tool in numerical studies. ${ }^{37,89}$

\subsubsection{Useful diagnosis parameters}

Insight into the geometric properties of MOTS worldtubes leads to useful diagnosis parameters for monitoring dynamical evolutions. Geometric black hole inequalities provide a particular avenue. In particular, the conjectured Penrose's inequality $A \leq 16 \pi M_{\mathrm{ADM}}^{2}$ for asymptotically flat spacetimes provides a bound to the $\mathrm{AH}$ area (strictly speaking, the bound is on the area of a minimal surface enclosing the $\mathrm{AH}$ ). A violation of $\epsilon_{\text {Penrose }} \equiv A /\left(16 \pi M_{\mathrm{ADM}}^{2}\right) \leq 1$ indicates a more exterior MOTS. In the axially symmetric case this can be refined in terms of a so-called ${ }^{90,91}$ Dain number

$$
\epsilon_{\text {Dain }} \equiv \frac{A}{8 \pi\left(M_{\mathrm{ADM}}^{2}+\sqrt{M_{\mathrm{ADM}}^{4}-J^{2}}\right)} \leq 1 .
$$

Moreover, the rigidity part of the conjecture provides an extremely simple characterization of Kerr as satisfying $\epsilon_{\text {Dain }}=1$. In the same spirit, the geometric inequality ${ }^{92}$ $J \leq M_{\text {ADM }}^{2}$ provides a characterization of (sub)extremality of black holes. However, these inequalities involve total quantities such as the ADM mass. It is remarkable that the dynamical horizon structure (actually the outer trapping horizon condition) provides exactly the needed conditions to prove the quasi-local inequality ${ }^{42,93,94}$

$$
A \geq 8 \pi|J|,
$$

in generic spacetimes with matter satisfying the dominant energy condition. The validity of the area-angular momentum inequality (60) is equivalent to the nonnegativity of the surface gravity $\kappa$ of isolated and dynamical horizons, ${ }^{32}$ supporting the internal consistency of their first law of black hole thermodynamics. Inequality (60) provides a quasi-local characterization of black hole (sub)extremality, that is directly related to changes in the horizon metric type ${ }^{80}$ and jumps discussed in Sec. 5.1.1. This is also the context of the Booth $\&$ Fairhurst extremality parameter $^{80,95}$

$$
e \equiv 1+\frac{1}{4 \pi} \int_{\mathcal{S}} d A \delta_{k} \theta^{(\ell)} \leq 1
$$

\subsection{Heuristic and effective approaches in a posteriori spacetime analysis}

Hitherto we have discussed analysis tools to be applied in numerically constructed spacetimes, but related to sound geometric structures. However, when developing a qualitative understanding of the underlying dynamics, involving, e.g. a comparison with Newtonian or Special Relativity scenarios, the available geometric notions are often not enough. This is manifest in astrophysical contexts requiring estimations 
for linear, orbital angular momentum or binding energies. In some cases, a choice must be done between saying nothing at all or rather adopting a heuristic approach.

An example of the latter is the following heuristic proposal ${ }^{96}$ for a quasi-local black hole linear momentum. Given a vector $\xi^{a}$ transverse to a MOTS $\mathcal{S}$, applying on $\mathcal{S}$ the linear momentum ADM prescription at spatial infinity leads to

$$
P(\xi)=\frac{1}{8 \pi} \int_{\mathcal{S}_{t}}\left(K_{a b}-K \gamma_{a b}\right) \xi^{a} s^{b 2} \epsilon
$$

In spite of its ad hoc nature, this quantity has been successfully applied in the analysis ${ }^{96}$ of linear and orbital angular momentum in binary black hole orbits and in the recoil dynamics of the black hole resulting of asymmetric binary mergers.

\subsection{An effective correlation approach to the analysis of spacetime dynamics}

The qualitative and quantitative understanding of strong-field spacetime dynamics represents a challenge in gravitational physics both at a fundamental level and in applications. In astrophysical settings a natural strategy consists in extending to general relativistic scenarios the Newtonian celestial mechanics approach. This has indeed led to fundamental achievements in the understanding of the physics of compact objects. However, the focus on the properties of individual objects, in particular in multi-component systems, also meets fundamental obstacles in a gravitational theory (i) without a priori rigid structures providing canonical structures, and (ii) with global aspects playing a crucial role. The latter encompasses global causal issues and also the in-built elliptic character of certain objects, both aspects relevant in the characterization of black holes. In this context, an approach to spacetime analysis that explicitly emphasizes the global/quasi-local properties of the relevant fields, at the price of renouncing to a detailed tracking of the geometry and trajectories of small compact regions, can offer complementary insights to the celestial mechanics approach. Such a coarse-grained effective description is much in the spirit of the correlation approach in the analysis of complex condensed-matter systems or in quantum/statistical-field theory, where the functional structure of the (local) dynamical fields is encoded in the associated $n$-point correlation functionals. ${ }^{\mathrm{h}}$ Such an approach underlines the relational aspects of the theory, as a complementary methodology to the isolation of the dynamical properties a compact parts of the system. In sum, we can paraphrase the strategy as aiming at a functional and coarse-grained description of the spacetime geometry, by importing functional tools for the analysis of condensed matter and quantum/statistical field theory systems.

\footnotetext{
${ }^{\mathrm{h}} N$-point correlation functions encode the functional structure of the local fields. A coarse-grained description appear as a truncation to a finite number of $n$-point functions.
} 


\subsubsection{Cross-correlations of geometric quantities at test screens}

The strategy outlined above is admittedly vague. We sketch now a particular implementation ${ }^{97}$ of some of its aspects in a cross-correlation approach to the analysis of spacetime dynamics. Aiming at studying the gravitational dynamics in a given spacetime region $\mathcal{R}$, we consider an outer $\mathcal{B}_{o}$ and an inner $\mathcal{B}_{i}$ hypersurfaces lying in the causal future of $\mathcal{R}$. These hypersurfaces are taken as outer and inner boundaries of the bulk spacetime region of interest. The geometry of $\mathcal{B}_{o}$ and $\mathcal{B}_{i}$ is causally affected by the dynamics in $\mathcal{R}$, so that $\mathcal{B}_{o}$ and $\mathcal{B}_{i}$ can be understood as balloon probes into the spacetime geometry. In other words, $\mathcal{B}_{o}$ and $\mathcal{B}_{i}$ provide test screens (they do not back-react on the bulk dynamics) on which we can construct geometric quantities $h_{o}$ and $h_{i}$ to be cross-correlated. Choosing causally disconnected screens $\mathcal{B}_{o}$ and $\mathcal{B}_{i}$, a nontrivial correlation between $h_{o}$ and $h_{i}$ encodes geometric information about the common past region $\mathcal{R}$. We can think of this as the reconstruction of the interaction region from the debris in a scattering experiment (inverse scattering picture). Let us now restrict ourselves to the study of near-horizon spacetime dynamics. ${ }^{97}$ In an (asymptotically flat) black hole spacetime setting, null infinity $\mathscr{I}^{+}$and the (event) black hole horizon $\mathscr{E}$ provide canonical choices for $\mathcal{B}_{o}$ and $\mathcal{B}_{i}$, respectively (cf. Fig. 3). Retarded and advanced null coordinates $u$ and $v$ provide good parameters for quantities $h_{o}$ and $h_{i}$ calculated as integrals on sections $\mathcal{S}_{u} \subset \mathscr{I}^{+}$ and $\mathcal{S}_{v} \subset \mathcal{E}$. A meaningful notion for the cross-correlation between $h_{o}(u)$ and $h_{i}(v)$, considered as time series, requires the introduction of a (gauge-dependent) mapping between $u$ and $v$ at $\mathscr{I}^{+}$and $\mathcal{E}$. We refer to this point as the time-stretching issue.

\subsubsection{Cross-correlations in an Initial Value Problem approach: Dynamical horizons as canonical inner probe screens}

The adopted Initial Value Problem approach has a direct impact in the crosscorrelation picture above. In particular, the event horizon is not available during the evolution. ${ }^{i}$ Instead, the (outermost) $\mathrm{DH} \mathcal{H}$ fixed by the chosen $3+1$ foliation stands as a natural spacetime inner boundary $\mathcal{B}_{i}$. Although any hypersurface covering the black hole singularity could be envisaged for the present cross-correlation purposes, the $\mathrm{DH} \mathcal{H}$ provides a natural geometric prescription. Regarding the timestretching issue, the time function $t$ defining the $3+1$ spacetime slicing automatically implements a (gauge) mapping between retarded and advanced times $u$ and $v$. Crosscorrelations between geometric quantities at $\mathcal{H}$ and $\mathscr{I}^{+}$can then be calculated as standard time-series $h_{i}(t)$ and $h_{o}(t)$ (cf. Fig. 4). Due to the gauge nature of $t$, the geometric information in quantities $h_{i}(t)$ and $h_{2}(t)$ is not encoded in their local (arbitrary) time dependence, but rather in the global structure of successive maxima and minima. The calculation of cross-correlations must take this into account. ${ }^{97}$ This means, in particular, that quantities to be correlated must be scalars.

\footnotetext{
${ }^{i}$ Regarding $\mathscr{I}^{+}$, a pragmatic choice in a Cauchy approach consists in substituting it by a timelike worldtube of large radii spheres. However, $\mathscr{I}^{+}$can be kept if using a hyperboloidal foliation.
} 


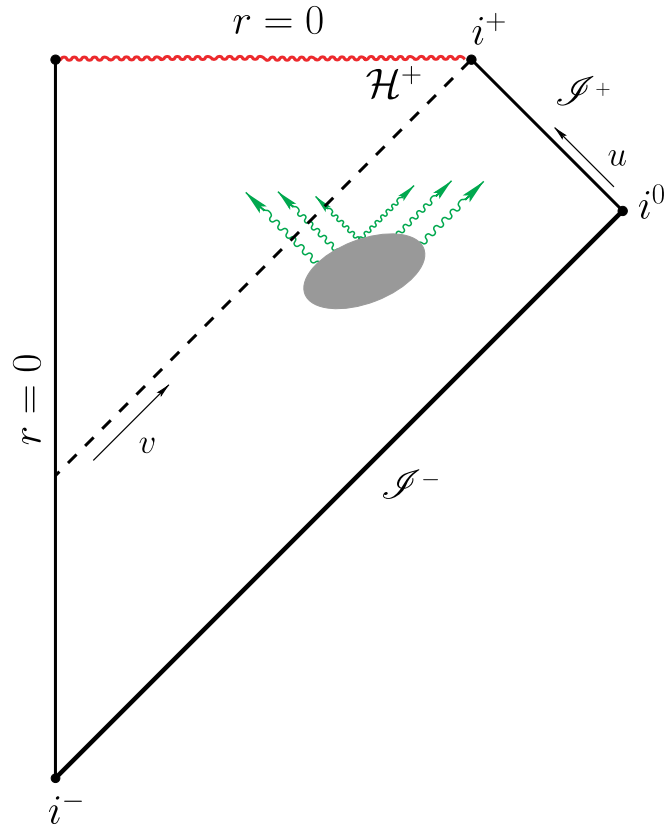

Fig. 3. (Color online) Carter-Penrose diagram representing a generic (spherically symmetric) collapse and illustrating the cross-correlation approach to near-horizon gravitational dynamics.

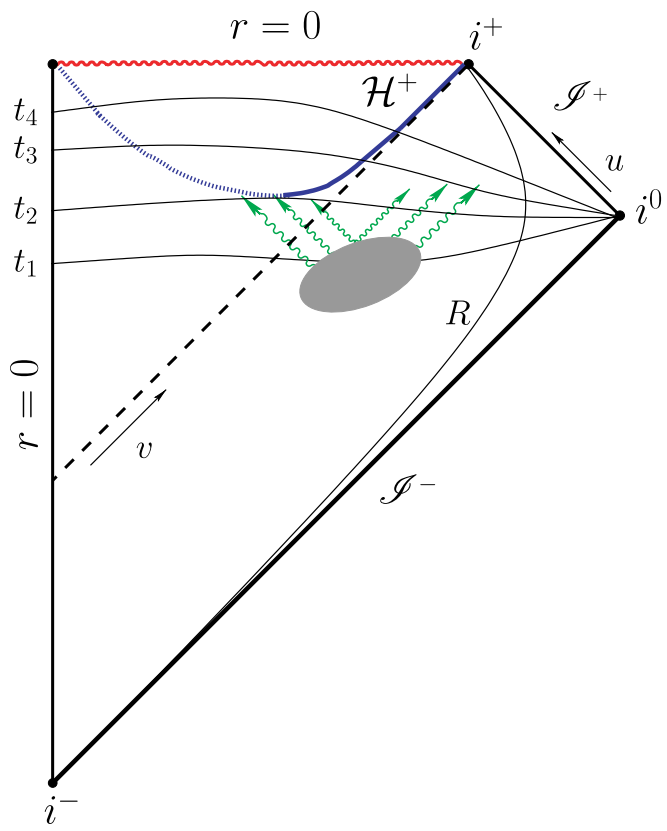

Fig. 4. (Color online) Carter-Penrose diagram for the cross-correlation picture in a Cauchy IVP approach. 


\subsubsection{Application to black hole recoil dynamics: Towards DH news functions}

In the context of the study of black hole recoil dynamics after an asymmetric merger, let us take $h_{o}(u)$ as the Bondi flux of linear momentum along a (preferred) direction

$$
\frac{d P^{\mathrm{B}}[\xi]}{d u}(u)=\lim _{(u, r \rightarrow \infty)} \frac{r^{2}}{8 \pi} \oint_{\mathcal{S}_{u, r}}\left(\xi^{i} s_{i}\right)|\mathcal{N}(u)|^{2} d \Omega, \quad \mathcal{N}(u)=\int_{-\infty}^{u} \Psi_{4}\left(u^{\prime}\right) d u^{\prime}
$$

Here $\mathcal{N}$ is the news function at $\mathscr{I}^{+}$, and $\xi^{a}$ is a given spacelike transverse direction to $\mathcal{S}_{u, r}$, so that $\left(d P^{\mathrm{B}} / d u\right)[\xi]$ is a scalar. A natural choice ${ }^{\mathrm{j}}$ for $h_{i}(v)$ would be given by the expression (63) with $\Psi_{4}$ at $\mathscr{I}^{+}$substituted by some $\Psi_{0}$ at $\mathcal{H}$. A preferred null tetrad on $\mathcal{S}_{v}$ is then needed, something that for DHs is provided by $\ell_{N}^{a}$ and $k_{N}^{a}$ in (41). Using them in (20), the preferred Weyl scalar $\Psi_{0}^{N}$ is employed to construct

$$
\tilde{K}^{N}[\xi](v)=-\frac{1}{8 \pi} \oint_{\mathcal{S}_{v}}\left(\xi^{i} s_{i}\right)\left|\tilde{\mathcal{N}}_{N}^{(0)}(v)\right|^{2} d A, \quad \text { with } \tilde{\mathcal{N}}_{N}^{(0)}(v)=\int_{v_{0}}^{v} \Psi_{0}^{N}\left(v^{\prime}\right) d v^{\prime}
$$

In spite of the formal similarity between (63) and (64) there is a fundamental difference: whereas $\left(d P^{\mathrm{B}} / d u\right)[\xi]$ is an instantaneous flux through $\mathscr{I}^{+}$, this is not true for $\tilde{K}^{N}[\xi](v)$. The function $\mathcal{N}(u)$ can be written in terms of geometric quantities on sections $\mathcal{S}_{u}$. This local-in-time behavior is a crucial feature of any valid news function and it is not shared by $\tilde{\mathcal{N}}_{N}^{(0)}(v)$. However, it suffices to modify $\tilde{\mathcal{N}}_{N}^{(0)}(v)$ with terms completing the integrand $\Psi_{0}^{N}\left(v^{\prime}\right)$ to a total differential in time. Noting $q_{a}^{c} q_{b}^{d} C_{l c f d} \ell^{l} \ell^{f}=\Psi_{0} \bar{m}_{a} \bar{m}_{b}+\bar{\Psi}_{0} m_{a} m_{b}$, inspection of Eq. (22) [actually its dynamical version with $h^{a}$ instead of $\ell^{a}$ ] suggests the identification of a correct news-like function at $\mathcal{H}$ as proportional to the shear $\sigma_{a b}^{(h)}$ (see also Refs. 99 and 100 for the discussion of the news in quasi-local contexts). In tensorial notation, we write

$$
\frac{d P^{N}}{d v}[\xi](v)=-\frac{1}{8 \pi} \oint_{\mathcal{S}_{v}}\left(\xi^{i} s_{i}\right)\left(\mathcal{N}_{a b}^{\mathrm{N}, \mathrm{g}} \mathcal{N}_{\mathrm{N}, \mathrm{g}}^{a b}\right) d A, \quad \text { with } \mathcal{N}_{a b}^{\mathrm{N}, \mathrm{g}}=-\frac{1}{\sqrt{2}} \sigma_{a b}^{(h)},
$$

where the coefficient in $\mathcal{N}_{a b}^{\mathrm{N}, \mathrm{g}}$ guarantees the correct factor in the leading-term. This $\left(d P^{N} / d v\right)[\xi]$ provides a natural quantity to be correlated with $\left(d P^{\mathrm{B}} / d u\right)[\xi]$. The notation underlines the local character in time as the flux of a quantity $P^{N}[\xi]$, but no physical meaning is given to the latter. It is worthwhile, though, to remark

${ }^{\text {j}}$ We also mention an effective curvature vector ${ }^{97,98}$ constructed from the Ricci scalar ${ }^{2} R$ on sections $\mathcal{S}_{v}$ of $\mathcal{H}$, that provides an intrinsic prescription for $h_{i}(v)$ leading to nontrivial ${ }^{97}$ cross-correlations with $\left(d P^{\mathrm{B}} / d u\right)[\xi]$. 
the formal similarity of the monopolar part of the square of the news $\mathcal{N}_{a b}^{\mathrm{N}, \mathrm{g}}$, i.e.

$$
\begin{aligned}
\frac{d E^{N}}{d v}(v) & =\frac{1}{16 \pi} \oint_{\mathcal{S}_{v}} \sigma_{a b}^{(h)} \sigma_{(h)}^{a b} d A \\
& =\frac{1}{16 \pi} \oint_{\mathcal{S}}\left[\sigma_{a b}^{(\ell)} \sigma^{(\ell)}{ }^{a b}-2 C \sigma_{a b}^{(\ell)} \sigma^{(k)^{a b}}+C^{2} \sigma_{a b}^{(k)} \sigma^{(k)^{a b}}\right] d A
\end{aligned}
$$

with the expression of the flux of gravitational energy 26,44 through a DH, in particular with its transverse part. ${ }^{45,46}$ The identification of $\sigma_{a b}^{(h)}$ as a news-like function suggests a further step, by introducing a heuristic notion of Bondi-like 4-momentum flux through $\mathcal{H}$. Considering the unit normal $\hat{\tau}^{a}$ to $\mathcal{H}\left(\hat{\tau}^{a}=\tau^{a} / \sqrt{\left|\tau^{b} \tau_{b}\right|}=\right.$ $\left.\left(\ell^{a}+C k^{a}\right) / \sqrt{2 C}=\left(b n^{a}+N s^{a}\right) / \sqrt{2 C}\right)$, and for a generic spacetime vector $\eta^{a}$

$$
\frac{d P_{\tau}^{N}}{d v}[\eta]=-\frac{1}{16 \pi} \oint_{\mathcal{S}_{v}}\left(\eta^{a} \hat{\tau}_{a}\right) \sigma_{a b}^{(h)} \sigma_{(h)}^{a b} d A
$$

has formally the expression of a Bondi-like 4-momentum. ${ }^{\mathrm{k}}$ The flux of energy associated with an Eulerian observer $n^{a}$ would be

$$
\frac{d E_{\tau}^{N}}{d v}(v) \equiv \frac{d P_{\tau}^{N}}{d v}\left[n^{a}\right]=\frac{1}{16 \pi} \oint_{\mathcal{S}} \frac{b}{\sqrt{2 C}}\left(\sigma_{a b}^{(h)} \sigma^{(h)^{a b}}\right) d A
$$

where $\frac{b}{\sqrt{2 C}}=\sqrt{1+N^{2} / 2 C}$. The flux of linear momentum for $\xi^{a} \in T \Sigma_{t}$ would be

$$
\frac{d P_{\tau}^{N}}{d v}[\xi]=-\frac{1}{16 \pi} \oint_{\mathcal{S}_{v}} \frac{N}{\sqrt{2 C}}\left(\xi^{a} s_{a}\right)\left(\sigma_{a b}^{(h)} \sigma^{(h)^{a b}}\right) d A .
$$

Near equilibrium $(C \rightarrow 0)$, we have $\sigma_{a b}^{(h)} \sigma_{(h)}^{a b} \sim C$ on DHs [cf. Eq. (17)] so that expressions (68) and (69) are regular $(O(\sqrt{C}))$. Integrating $(69)$ in time would lead to a Bondi-like counterpart ${ }^{1}$ of the heuristic $A D M$-like linear momentum in (62).

Before finishing this section, let us mention that the present discussion on horizon news-like functions can be related ${ }^{97}$ to a viscous fluid analogy for quasi-local horizons. ${ }^{23,51}$ In particular, geometric decay and oscillation timescales (respectively, $\tau$ and $T$ ) can be constructed on the horizon ${ }^{97}$ from the expansion $\theta^{(h)}$ and shear $\sigma_{a b}^{(h)}$, respectively related to bulk and shear viscosity terms. In the context of black hole recoil dynamics, this provides an instantaneous geometric prescription for a slowness parameter ${ }^{101} P=T / \tau$ controlling the qualitative aspects of the dynamics.

\footnotetext{
${ }^{\mathrm{k}}$ An alternative expression would follow by using in (67), instead of $\sigma_{a b}^{(h)} \sigma^{(h)^{a b}}$, the integrand in the DH energy flux, ${ }^{26,44-46}$ that would also include the longitudinal part $\Omega_{a}^{(\ell)} \Omega^{(\ell)}{ }^{a}$.

${ }^{1} \mathrm{~A}$ related prescription for a DH linear momentum flux would be given by angular integration of the appropriate components in the effective gravitational-radiation energy-tensor of Ref. 46.
} 


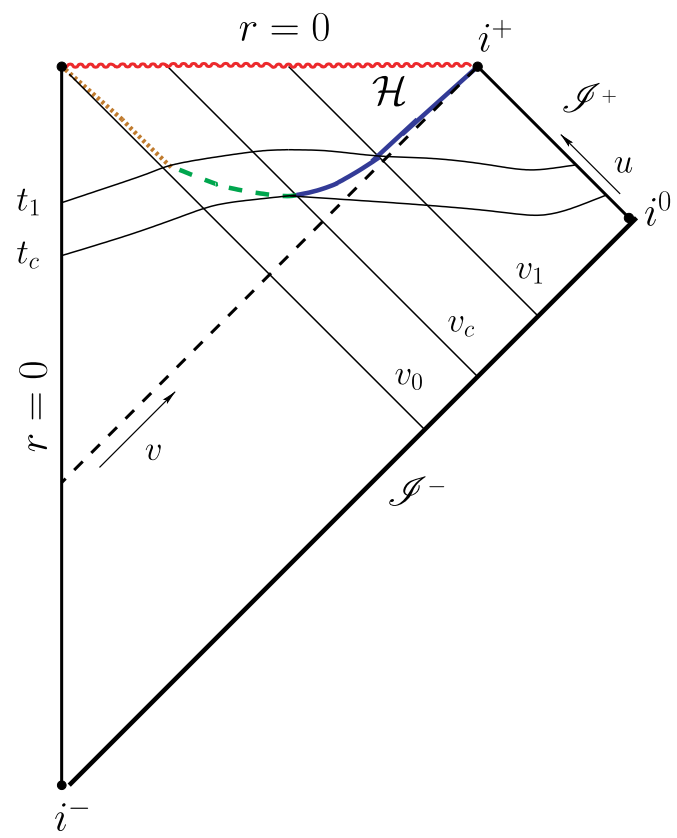

Fig. 5. (Color online) Illustration of the splitting of a DH into internal and external sections by a $3+1$ slicing.

\subsubsection{The role of the inner horizon in the integration of fluxes along $\mathcal{H}$}

Flux integrations along $\mathcal{H}$ require appropriate parametrizations of $\mathcal{H}$, such as an advanced time $v$. Then, given the flux $F_{Q}(v)$ of a quantity $Q(v)$, we can write ${ }^{\mathrm{m}}$

$$
Q(v)=Q\left(v_{0}\right)+\operatorname{sign}(C) \int_{v_{0}}^{v} F_{Q}\left(v^{\prime}\right) d v^{\prime},
$$

this requiring an initial value $Q\left(v_{0}\right)$. However, such coordinate $v$ is not natural in an Initial Value Problem approach. As discussed in Sec. 5.1.1, the $3+1$ slicing $\left\{\Sigma_{t}\right\}$ induces a splitting of the DH into internal and external sections. The integration in (70) can then be split into external and internal horizon parts (cf. Fig. 5)

$Q(t)=Q\left(v_{0}\right)+\operatorname{sign}(C) \int_{t_{c}}^{t}\left(F_{Q}\right)^{\mathrm{int}}\left(t^{\prime}\right) d t^{\prime}+\operatorname{sign}(C) \int_{t_{c}}^{t}\left(F_{Q}\right)^{\operatorname{ext}}\left(t^{\prime}\right) d t^{\prime}+\operatorname{Res}(t)$,

where the error $\operatorname{Res}(t)$ is $\operatorname{Res}(t)=\operatorname{sign}(C) \int_{t}^{\infty}\left(F_{Q}\right)^{\operatorname{int}}\left(t^{\prime}\right) d t^{\prime}$.

If the growth of $Q$ is understood as ultimately associated with some flow into the black hole singularity, the actual essential role of the horizon $\mathcal{H}$ would be that of capturing the associated fluxes. This assumes that the worldtube $\mathcal{H}$ begins at the formation of the singularity. More complex singularity structures (as those coming

${ }^{m}$ The coefficient $\operatorname{sign}(C),+1$ for spacelike $\mathcal{H}$ and -1 for timelike $\mathcal{H}$, takes into account the possible integration of fluxes happening when timelike sections of $\mathcal{H}$ occur; cf. Fig. 2. 
from a binary merger) would require a more detailed analysis of this point. From this perspective, there is nothing intrinsically special about dynamical horizons: any hypersurface separating the black hole singularity from past null infinity $\mathscr{I}^{-}$(e.g. the event horizon) would be appropriate for fluxes evaluation. However, from a quasi-local perspective, if DHs are shown to cover systematically the black hole singularity (or, more generally, the inner Cauchy horizon), they actually provide excellent geometric prescriptions for such test screens (this is the motivation for the point (iii) in Sec. 3.2.6).

\subsubsection{Auxiliary test-field evolutions in curved backgrounds}

In Sec. 5.4.3 we have considered cross-correlations between different contractions of the Weyl tensor at distinct hypersurfaces. It is legitimate to question if such crosscorrelations are meaningful at all, given the a priori different geometric content of the involved functions. Let us consider the following approach to this issue: evolve, together with the gravitational degrees of freedom in Einstein equations, an auxiliary (set of) scalar field(s) $\Phi_{i}$ without back-reaction on the geometry (i.e. test fields) and whose evolution on the dynamically evolving background spacetime closely tracks $^{\mathrm{n}}$ its relevant geometric features. Then, the correlation approach outlined in Subsec. 5.4 for a (coarse-grained) extraction of geometric content, can be applied directly on $\Phi_{i}$. We can paraphrase this approach as pouring sand on a transparent surface. On the one hand, this removes the ambiguity in the choice of quantities $h_{i}$ and $h_{o}$ at inner and outer hypersurfaces. On the other hand, and more importantly, it also permits to extend to the bulk spacetime the (cross-) correlation strategy between spacetime boundaries.

\section{General Perspective}

We have presented an introduction to some aspects of quasi-local black holes in an Initial Value Problem approach to the spacetime construction. From a fundamental perspective, quasi-local black hole horizons provide crucial insights into the geometry of the black hole and trapped regions and a sound avenue to black hole physics in generic scenarios. However, quasi-local black holes also meet challenges when considered as physical surfaces of a compact object. We have adopted a pragmatic or effective approach in which quasi-local black hole horizons are understood as hypersurfaces with remarkable geometric properties that provide worldtubes of canonical surfaces in a given $3+1$ slicing of the spacetime. We have shown how they can be used as an a priori ingredient in evolution schemes to Einstein equations, where they provide inner boundary conditions for black hole spacetimes. Then we have

\footnotetext{
${ }^{\mathrm{n}}$ See Ref. 102 for a discussion of a similar approach in a binary black hole context, and Ref. 35 for a methodology sharing part of the spirit but directly tracking spacetime curvature quantities.
} 
illustrated their use as a posteriori analysis tools tracking and characterizing quasilocally the black hole properties and providing, through their rigidity properties, excellent test-screen probes into the near-horizon black hole spacetime geometry.

\section{Acknowledgments}

I thank the organizers of the 2011 Shanghai Asia-Pacific School, especially C.-M. Cheng, S. A. Hayward and J. Nester, for their kind invitation and hospitality. I thank E. Gourgoulhon, B. Krishnan, R. P. Macedo, P. Mösta, A. Nielsen and L. Rezzolla for the interactions during the writing of these notes. I acknowledge the support of the Alexander von Humboldt Foundation, the Spanish MICINN (FIS2008-06078C03-01) and the Junta de Andalucía (FQM2288/219).

\section{References}

1. R. Penrose, Annals N. Y. Acad. Sci. 224 (1973) 125.

2. R. Penrose, Phys. Rev. Lett. 14 (1965) 57.

3. S. Hawking, Proc. R. Soc. London, Ser. A 300 (1967) 182.

4. S. Hawking and R. Penrose, Proc. R. Soc. London, Ser. A 314 (1970) 529.

5. S. W. Hawking and G. F. R. Ellis, The Large Scale Structure of Space-Time (Cambridge University Press, 1973).

6. R. Penrose, Riv. Nuovo Cim. 1 (1969) 252.

7. M. Heusler, Liv. Rev. Relat. 1 (1998) 6.

8. S. W. Hawking, Phys. Rev. Lett. 26 (1971) 1344.

9. S. W. Hawking, Commun. Math. Phys. 25 (1972) 152.

10. J. M. Bardeen, B. Carter and S. W. Hawking, Commun. Math. Phys. 31 (1973) 161.

11. V. Faraoni and A. B. Nielsen, arXiv:1103.2089.

12. M. Kriele and S. A. Hayward, J. Math. Phys. 38 (1997) 1593.

13. P. T. Chruściel, in The Conformal Structure of Spacetime: Geometry, Analysis, Numerics, J. Frauendiener and H. Friedrich (eds.), Lecture Notes in Physics (Springer, 2002), p. 61.

14. S. Hayward, Phys. Rev. D 49 (1994) 6467.

15. J. M. M. Senovilla, Int. J. Mod. Phys. D 22 (2011) 2139.

16. A. B. Nielsen, Class. Quant. Grav. 27 (2010) 245016.

17. D. M. Eardley, Phys. Rev. D 57 (1998) 2299.

18. E. Schnetter and B. Krishnan, Phys. Rev. D73 (2006) 021502.

19. I. Ben-Dov, Phys. Rev. D 75 (2007) 064007.

20. J. E. Aman, I. Bengtsson and J. M. M. Senovilla, J. Phys. Conf. Ser. 229 (2010) 012004.

21. I. Bengtsson and J. M. M. Senovilla, (2010) arXiv:1009.0225.

22. A. B. Nielsen, Int. J. Mod. Phys. D 22 (2011) 2205.

23. E. Gourgoulhon, Phys. Rev. D 72 (2005) 104007.

24. I. Booth and S. Fairhurst, Phys. Rev. D 75 (2007) 084019.

25. I. Racz, Class. Quant. Grav. 25 (2008) 162001.

26. A. Ashtekar and B. Krishnan, Phys. Rev. D 68 (2003) 104030.

27. I. Booth, Can. J. Phys. 83 (2005) 1073.

28. E. Gourgoulhon and J. L. Jaramillo, Phys. Rep. 423 (2006) 159.

29. B. Krishnan, Class. Quant. Grav. 25 (2008) 114005.

30. L. Andersson, M. Mars and W. Simon, Phys. Rev. Lett. 95 (2005) 111102. 
31. A. Ashtekar, C. Beetle and J. Lewandowski, Phys. Rev. D 64 (2001) 044016.

32. A. Ashtekar and B. Krishnan, Liv. Rev. Relat. 7 (2004) 10.

33. A. Ashtekar, C. Beetle and J. Lewandowski, Class. Quant. Grav. 19 (2002) 1195.

34. R. Penrose and W. Rindler, Spinors and Space-Time, Vol. 1: Two-Spinor Calculus and Relativistic Fields (Cambridge University Press, 1984).

35. R. Owen, J. Brink, Y. Chen, J. D. Kaplan, G. Lovelace, K. D. Matthews, D. A. Nichols, M. A. Scheel, F. Zhang, A. Zimmerman and K. S. Thorne, Phys. Rev. Lett. 106 (2011) 151101.

36. A. Ashtekar, J. Engle, T. Pawlowski and C. Van Den Broeck, Class. Quant. Grav. 21 (2004) 2549.

37. E. Schnetter, B. Krishnan and F. Beyer, Phys. Rev. D 74 (2006) 024028.

38. M. Jasiulek, Class. Quant. Grav. 26 (2009) 245008.

39. R. Owen, Phys. Rev. D 80 (2009) 084012.

40. A. Ashtekar and G. J. Galloway, Adv. Theor. Math. Phys. 9 (2005) 1.

41. L. Andersson, M. Mars and W. Simon, Adv. Theor. Math. Phys. 12 (2008) 853.

42. J. L. Jaramillo, M. Reiris and S. Dain, arXiv: 1106.3743.

43. L. M. Cao, JHEP 03 (2011) 112.

44. A. Ashtekar and B. Krishnan, Phys. Rev. Lett. 89 (2002) 261101.

45. S. Hayward, Phys. Rev. Lett. 93 (2004) 251101.

46. S. A. Hayward, Phys. Rev. D 70 (2004) 104027.

47. I. Booth and S. Fairhurst, Phys. Rev. Lett. 92 (2004) 011102.

48. S. A. Hayward, arXiv:0810.0923.

49. Y. H. Wu and C. H. Wang, Phys. Rev. D 80 (2009) 063002.

50. Y. H. Wu and C. H. Wang, Phys. Rev. D 83 (2011) 084044.

51. E. Gourgoulhon and J. L. Jaramillo, Phys. Rev. D 74 (2006) 087502.

52. E. Gourgoulhon and J. L. Jaramillo, New Astron. Rev. 51 (2008) 791.

53. S. W. Hawking and J. B. Hartle, Commun. Math. Phys. 27 (1972) 283.

54. S. A. Hayward, Phys. Rev. D 74 (2006) 104013.

55. S. A. Hayward, arXiv:gr-qc/0607081.

56. I. Booth, M. P. Heller and M. Spalinski, Phys. Rev. D 83 (2011) 061901.

57. A. B. Nielsen, M. Jasiulek, B. Krishnan and E. Schnetter, Phys. Rev. D 83 (2011) 124022 .

58. I. Booth, M. P. Heller, G. Plewa and M. Spalinski, Phys. Rev. D 83 (2011) 106005.

59. C. Williams, Annales Henri Poincare 9 (2008) 1029.

60. C. Williams, Commun. Math. Phys. 293 (2010) 589.

61. C. Williams, arXiv:1005.5401.

62. H. Friedrich, Ann. Phys. (Berlin) 15 (2005) 84.

63. J. L. Jaramillo, J. A. Valiente Kroon and E. Gourgoulhon, Class. Quant. Grav. 25 (2008) 093001.

64. E. Gourgoulhon, Lectures delivered at Institut Henri Poincaré in 2006, arXiv:grqc/0703035.

65. A. Lichnerowicz, J. Math. Pures Appl. 23 (1944) 37. Reprinted in A. Lichnerowicz, Choix d'œuvres mathématiques (Hermann, Paris, 1982).

66. J. W. York Jr., Phys. Rev. Lett. 82 (1999) 1350.

67. H. P. Pfeiffer and J. W. York Jr., Phys. Rev. D 67 (2003) 044022.

68. S. Bonazzola, E. Gourgoulhon, P. Grandclément and J. Novak, Phys. Rev. D 70 (2004) 104007.

69. I. Cordero-Carrion, J. M. Ibanez, E. Gourgoulhon, J. L. Jaramillo and J. Novak, Phys. Rev. D 77 (2008) 084007.

70. I. Cordero-Carrion et al., Phys. Rev. D 79 (2009) 024017. 
71. D. Gannon, J. Math. Phys. 16 (1975) 2364.

72. D. Gannon, Gen. Rel. Grav. 7 (1976) 219.

73. J. L. Jaramillo and E. Gourgoulhon and G. A. Mena Marugán, Phys. Rev. D 70 (2004) 124036.

74. G. B. Cook and H. P. Pfeiffer, Phys. Rev. D 70 (2004) 104016.

75. S. Dain, J. L. Jaramillo and B. Krishnan, Phys. Rev. D 71 (2005) 064003.

76. J. L. Jaramillo, M. Ansorg and F. Limousin, Phys. Rev. D 75 (2007) 024019.

77. J. L. Jaramillo, Phys. Rev. D 79 (2009) 087506.

78. A. B. Nielsen and M. Visser, Class. Quant. Grav. 23 (2006) 4637.

79. I. Booth, L. Brits, J. A. Gonzalez and C. Van Den Broeck, Class. Quant. Grav. 23 (2006) 413.

80. I. Booth and S. Fairhurst, Phys. Rev. D77 (2008) 084005.

81. J. L. Jaramillo, E. Gourgoulhon, I. Cordero-Carrion and J. M. Ibanez, Phys. Rev. D 77 (2008) 047501.

82. J. Thornburg, Liv. Rev. Relat. 10 (2007) 3.

83. J. L. Jaramillo, M. Ansorg and N. Vasset, AIP Conf. Proc. 1122 (2009) 308.

84. L. B. Szabados, Liv. Rev. Relat. 12 (2009) 4.

85. J. L. Jaramillo and E. Gourgoulhon, Mass and Angular Momentum in General Relativity, Springer Series, Fundamental Theories of Physics (Springer, 2010).

86. O. Dreyer, B. Krishnan, D. Shoemaker and E. Schnetter, Phys. Rev. D 67 (2003) 024018.

87. G. B. Cook and B. F. Whiting, Phys. Rev. D 76 (2007) 041501.

88. M. Korzynski, Class. Quant. Grav. 24 (5944) 5935.

89. N. Vasset, J. Novak and J. L. Jaramillo, Phys. Rev. D 79 (2009) 124010.

90. S. Dain, C. O. Lousto and R. Takahashi, Phys. Rev. D 65 (2002) 104038.

91. J. L. Jaramillo, N. Vasset and M. Ansorg, EAS Publications Series 30 (2008) 257.

92. S. Dain, Phys. Rev. Lett. 96 (2006) 101101.

93. J. Hennig, C. Cederbaum and M. Ansorg, Commun. Math. Phys. 293 (2010) 449.

94. S. Dain and M. Reiris, arXiv:1102.5215.

95. I. Booth, arXiv:0709.0934.

96. B. Krishnan, C. O. Lousto and Y. Zlochower, Phys. Rev. D 76 (2007) 081501.

97. J. L. Jaramillo, R. P. Macedo, P. Moesta and L. Rezzolla, arXiv:1108.0060, arXiv:1108.0061.

98. L. Rezzolla, R. P. Macedo and J. L. Jaramillo, Phys. Rev. Lett. 104 (2010) 221101.

99. S. A. Hayward, Class. Quantum Grav. 11 (1994) 3037.

100. S. A. Hayward, Phys. Rev. D 68 (2003) 104015.

101. R. H. Price, G. Khanna and S. A. Hughes, Phys. Rev. D 83 (2011) 124002.

102. E. Bentivegna, D. M. Shoemaker, I. Hinder and F. Herrmann, Phys. Rev. D 77 (2008) 124016. 